\title{
DUST EXTINCTION OF THE STELLAR CONTINUA IN STARBURST GALAXIES: THE ULTRAVIOLET AND OPTICAL EXTINCTION LAW
}

\author{
Daniela Calzetti and ANNe L. KinNeY ${ }^{1,2}$ \\ Space Telescope Science Institute, 3700 San Martin Drive, Baltimore MD 21218 \\ AND \\ Thaisa StORCHI-BeRgMANN ${ }^{2}$ \\ Instituto di Fisica, Universidade Federal Rio Grande do Sul, Brazil \\ Received 1993 August 6; accepted 1994 January 14
}

\begin{abstract}
We analyze the IUE UV and the optical spectra of 39 starburst and blue compact galaxies in order to study the average properties of dust extinction in extended regions of galaxies. The optical spectra have been obtained using an aperture which matches that of $I U E$, so comparable regions within each galaxy are sampled.

The data from the 39 galaxies are compared with five models for the geometrical distribution of dust, adopting as extinction laws both the Milky Way and the Large Magellanic Cloud laws. The commonly used uniform dust screen is included among the models. We find that none of the five models is in satisfactory agreement with the data.

In order to understand the discrepancy between the data and the models, we have derived an extinction law directly from the data in the UV and optical wavelength range. The resulting curve is characterized by an overall slope which is more gray than the Milky Way extinction law's slope, and by the absence of the $2175 \AA$ dust feature. Remarkably, the difference in optical depth between the Balmer emission lines $\mathrm{H} \alpha$ and $\mathrm{H} \beta$ is about a factor of 2 larger than the difference in the optical depth between the continuum underlying the two Balmer lines. We interpret this discrepancy as a consequence of the fact that the hot ionizing stars are associated with dustier regions than the cold stellar population is.

The absence of the $2175 \AA$ dust feature can be due either to the effects of the scattering and clumpiness of the dust or to a chemical composition different from that of the Milky Way dust grains. Disentangling the two interpretations is not easy because of the complexity of the spatial distribution of the emitting regions.

The extinction law of UV and optical spectral continua of extended regions can be applied to the spectra of medium- and high-redshift galaxies, where extended regions of a galaxy are, by necessity, sampled.

Subject headings: dust, extinction - galaxies: ISM - galaxies: photometry — galaxies: starburst ultraviolet: galaxies
\end{abstract}

\section{INTRODUCTION}

The study of the intrinsic spectral energy distribution (SED) of galaxies relies on removal of the effects of dust extinction from the observed SED. However, the effective dust extinction on the emerging radiation is one of the least understood of the physical phenomena which take place in a galaxy.

The difficulties in drawing a consistent picture of the dust extinction in extended regions (galaxies or other objects, such as $\mathrm{H}$ II regions) can be summarized into two main points: (1) the chemical and physical properties, such as composition and grain size distribution, of the dust in the interstellar medium (ISM) are not well determined; (2) the emerging fiux from an extended region is sensitive to the geometrical distribution of the dust inside the region.

From the early work of Mathis, Rumpl, \& Nordsiek (1977), many efforts have been devoted to the understanding of the composition and optical properties of the dust grains which

\footnotetext{
${ }^{1}$ Visiting Astronomer, Kitt Peak National Observatory, National Optical Astronomy Observatories, which is operated by AURA, Inc., under a cooperative agreement with the National Science Foundation.

2 Visiting Astronomer, Cerro Tololo Interamerican Observatory, National Optical Astronomy Observatories, which is operated by AURA, Inc., under a cooperative agreement with the National Science Foundation.
}

give origin to the Milky Way extinction law (see Fig. 1, where the curve is from the parameterization of Seaton 1979 and Howarth 1983). To name a few, Greenberg \& Chlewicky (1983), Draine \& Lee (1984), Fitzpatrick \& Massa $(1986,1988)$ and Désert, Boulanger, \& Pouget (1990) have studied different chemical mixtures in deriving the dust composition which cause the complex shape of the Milky Way extinction law. No definite agreement exists yet.

The situation is complicated when the data on the other two known extinction curves, the Large Magellanic Cloud (LMC) law and the Small Magellanic Cloud (SMC) law, are considered (Fig. 1; Koornneef \& Code 1981; Bouchet et al. 1985; see also Fitzpatrick 1989). The Milky Way, LMC, and SMC extinction laws have different trends as a function of the wavelength for $\lambda \lesssim 2600 \AA$ : the absolute value of the slope from the near to the far-UV increases and the $2175 \AA$ dust feature decreases in strength going from the Milky Way, to the LMC, to the SMC. This difference has been tentatively attributed to the different metallicity of the three galaxies [see Lequeux 1988 , where $\mathrm{O} / \mathrm{H}=12+\log (\mathrm{O} / \mathrm{H})=8.76,8.50$, and 8.00 for Milky Way, LMC, and SMC, respectively], and, therefore, to a different composition of the dust. Bromage \& Nandy (1983) and Pei (1992) propose that the difference in shape of the Milky Way, LMC, and SMC extinction laws is due to a diminishing 


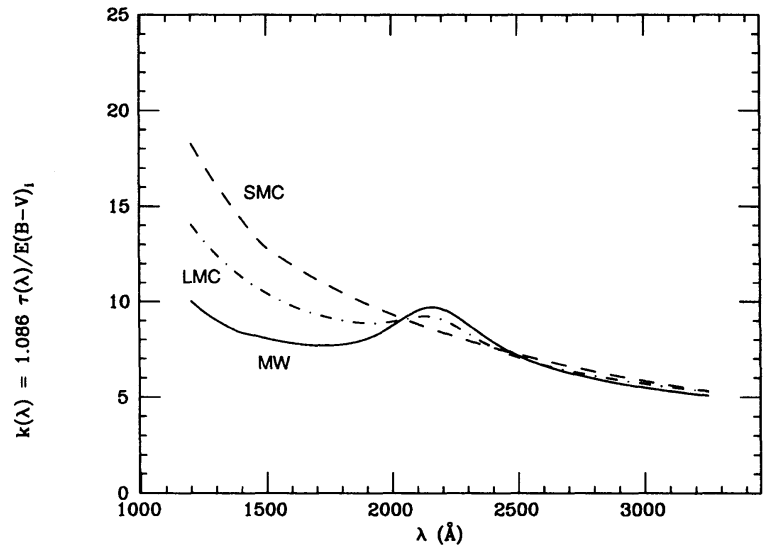

Fig. 1.-The extinction law $k(\lambda)$ is shown as a function of $\lambda$ for the Milky Way (continuous line), Large Magellanic Cloud (dot-dashed line), and Small Magellanic Cloud (dashed line). $k(\lambda)=A(\lambda) / E(B-V)_{i}=1.086 \tau(\lambda) / E(B-V)_{i}$, where $A(\lambda)$ is the extinction in magnitudes, $\tau(\lambda)$ is the extinction optical depth, and $E(B-V)_{i}$ is the color excess in $B$ relative to $V, A(V) / E(B-V)_{i}=3.1$.

value of the abundance ratio of graphite to silicates, the two dust grain types usually adopted in describing the Milky Way law (Mathis et al. 1977).

The problem of the geometrical distribution of dust (point [2] above) is strictly linked to the methodology used to derive extinction curves. Historically, the absolute value and the wavelength dependence of the dust extinction curve have been determined from the comparison between reddened and unreddened stars. Dealing with stars means that the dust between the observer and the target removes photons from the flux reducing the observed intensity: it is straightforward to assume in this case that the dust forms a uniform extinction screen between the star and the observer. In this configuration, the absorption and the scattering of photons by dust grains concur in removing flux from the line of sight.

However, when it comes to galaxies or, more generally, to extended regions, we are dealing with complex geometries for which the approximation of an extinction dust screen is insufficient. Dust can be mixed with the gas in the interstellar space (internal dust) and can be clumped. In addition, the scattering of photons by dust grains, which mostly contributes, together with the absorption, in removing photons from the line of sight, becomes effective at reconveying photons along the line of sight in the case of complex geometries for the dust configuration. A detailed analysis of the effects of scattering on the emerging flux from a plane parallel slab of homogeneously mixed ionized gas and dust has been presented by Bruzual, Magris, \& Calvet (1988). A wider approach to the problem has been discussed by Witt, Thronson, \& Capuano (1992), where alterations of the emerging broad-band SEDs are studied for different configurations of stars and dust. In both cases the main conclusion is that the application of the standard reddening curves inevitably leads to an overcorrection of the emerging fluxes. The interpretation is that, since heavily reddened stars are also heavily extincted, their contribution to the emerging flux is small in comparison to the contribution of less extincted stars. This interpretation is in line with the conclusions by Disney, Davies, \& Phillips (1989) that an inadequate treatment of the dust geometry can lead to incorrect evaluations of galaxy luminosities and star formation rates. However, as noted by Witt et al. (1992), the extinction corrections to be applied in the case of real galaxies are not straight- forward, since the detailed results from the various models strongly depend on the adopted geometry.

Geometrical effects of the dust distribution have also been invoked to explain the fact that in $\mathrm{H}$ II regions, which are extended regions although less complex than galaxies, the extinction measured from the Balmer line ratio $\mathrm{H} \alpha / \mathbf{H} \beta$ is lower than the extinction obtained by comparing $\mathrm{H} \beta$ with the nebular free-free radio emission (Sarazin 1976; Israel \& Kennicutt 1980; Lequeux et al. 1981; Caplan \& Deharveng 1986; Viallefond \& Goss 1986; van der Hulst et al. 1988; Skillman \& Israel 1988). Mathis (1983), Natta \& Panagia (1984), and Caplan \& Deharveng (1986) have discussed various models for the dust spatial distribution to explain the discrepancy; the conclusions favor models in which the positive contribution of scattering to the emerging flux is important and in which dust is internal or constitutes an external patchy foreground. Once more, details of the single $\mathrm{H}$ II region analyzed are important in defining the geometrical model of dust to be applied in each case.

Similar to the discrepancy between the extinctions derived from the Balmer lines and from the radio emission observed in $\mathrm{H}$ II regions, it has been noticed that the value of the extinction obtained from UV continuum of starburst galaxies is lower than the value obtained from the Balmer lines (cf. Fanelli, O'Connell, \& Thuan 1988; Keel 1993). Systematic studies devoted to understanding in detail the origin of the discrepancy have been not pursued yet (see, however, Keel 1993) because of the intrinsic difficulties in distinguishing the two contributions of the dust extinction and of the aging of the stellar population in the UV wavelength region. In fact, while the determination of the extinction in the optical region is based on the Balmer line ratios, which is sensitive only to the presence of dust (Osterbrock 1989), no such set of lines exists in the UV region (1220-3200 $\AA$ ).

The discrepancy between the extinction values obtained from the UV continuum and from the Balmer line ratio is not the only peculiarity of the UV spectra of extended extragalactic star-forming regions; a more puzzling characteristic is the weakness, and often absence, of the $2175 \AA$ dust feature, even in those cases where the value of the metallicity would indicate a Milky Way-like environment (Keel 1987; see also Rosa, Joubert, \& Benvenuti 1984 and Kinney et al. 1993).

In this paper we investigate the nature of and quantify the discrepancy between the dust extinction measured from the Balmer line ratios and from the UV stellar continuum, by analyzing the UV and optical spectra of a sample of the central regions of 39 line-emitting starburst and blue compact galaxies. Any a priori hypothesis on the shape of the extinction curve is avoided by studying the sample of galaxies from a statistical point of view. We derive an effective extinction law which can be applied to deredden UV and optical spectra of extended regions.

The UV spectra of the 39 galaxies are IUE spectra contained in the Atlas of Ultraviolet Spectra of Star-Forming Galaxies by Kinney et al. (1993). The optical spectra of the galaxies in our sample have been observed as part of a campaign to obtain optical spectra at KPNO and CTIO in an IUE-matched aperture of the 143 galaxies contained in the atlas.

The structure of the paper is as follows: $\S 2$ presents the observations and the initial analysis of the spectra; $\S 3$ discusses the correlation between optical and UV parameters of the spectra and relates the correlation to dust extinction; $\S 4$ introduces five models for the dust configuration, and the 
models are compared with the data in $\S 5$; in $\S 6$ the UV and optical effective extinction law for galaxies is derived; $\S 7$ contains the summary and the conclusions.

\section{OBSERVATIONS AND DATA ANALYSIS}

The UV spectra of the 39 starburst and blue compact galaxies in our sample are archival IUE spectra collected in the atlas by Kinney et al. (1993). The atlas spectra have been obtained by applying the optimal extraction technique on the archival $I U E$ line-by-line data files. This technique minimizes the noise in the final spectra (Kinney, Bohlin, \& Neill 1991).

The optical spectra of the galaxies have been obtained during five observing runs at KPNO and at CTIO, using apertures as close as possible to the $10^{\prime \prime} \times 20^{\prime \prime}$ aperture of IUE. Matched apertures have been chosen so that comparable regions of each galaxy in the UV and in the optical are sampled. Therefore, the information obtained from the UV and the optical spectra of our galaxies are likely to be selfconsistent.

The spectra of 19 galaxies in the sample (marked as $\mathrm{K}$ in the first column of Table 1) were observed during 1991 September and 1992 July with the KPNO $0.9 \mathrm{~m}+$ IRS, using a circular aperture of 13..5 diameter. The difference in flux due to the difference in size between the KPNO and the $I U E$ apertures is at most $30 \%$, which is within the uncertainty of the absolute optical fluxes. The spectra cover the wavelength range 3200 $7700 \AA$ with a resolution of $10 \AA$ (for more details on the observations, see McQuade, Kinney, \& Calzetti, 1994).

The optical spectra of the remaining 20 galaxies (marked as $\mathrm{C}$ in the first column of Table 1) were obtained during 1991 October-November, 1992 May, and 1992 November at the CTIO $1 \mathrm{~m}+2 \mathrm{D}$ Frutti and at the $1.5 \mathrm{~m}+\mathrm{CCD}$. A long slit

TABLE 1

Physical Properties of the SAMPLe

\begin{tabular}{|c|c|c|c|c|c|c|c|c|c|}
\hline $\begin{array}{l}\text { Observatory } \\
\text { (a) }\end{array}$ & $\begin{array}{c}\text { IUE } \\
\text { (b) }\end{array}$ & $\begin{array}{l}\text { Galaxy } \\
\text { Name }\end{array}$ & $\begin{array}{c}\text { Type } \\
\text { (c) }\end{array}$ & $\begin{array}{c}z \\
(d)\end{array}$ & $\begin{array}{c}\mathrm{E}(\mathrm{B}-\mathrm{V})_{G} \\
(\mathrm{e})\end{array}$ & $\begin{array}{c}\mathrm{EW}_{a b b} \\
\AA\end{array}$ & $\begin{array}{l}O / H \\
(\mathrm{f})\end{array}$ & $\begin{array}{c}\mathrm{E}(\mathrm{B}-\mathrm{V})_{i} \\
(\mathrm{~g})\end{array}$ & $\begin{array}{c}\tau_{B}^{l} \\
(\mathrm{~h})\end{array}$ \\
\hline $\mathrm{K}$ & S & IC1586 & BCG & 0.02 & 0.02 & 1.4 & 8.53 & 0.58 & $0.62-0.71 \pm 0.08$ \\
\hline $\mathrm{C}$ & S & Haro15 & BCG & 0.0214 & 0.02 & 5.6 & 8.57 & 0.00 & $0.00-1.33 \pm 0.06$ \\
\hline $\mathrm{K}$ & S & Mrk357 & SB & 0.053 & 0.04 & 4.2 & 8.53 & 0.12 & $0.13-1.19 \pm 0.08$ \\
\hline K & $\mathrm{S}$ & IC214 & SB & 0.0317 & 0.03 & 4.3 & 8.68 & 0.53 & $0.57-0.26 \pm 0.13$ \\
\hline $\mathrm{C}$ & $\mathrm{S}$ & NGC1140 & BCG & 0.0050 & 0.03 & 4.6 & 8.51 & 0.10 & $0.11-1.64 \pm 0.04$ \\
\hline $\mathrm{C}$ & $\mathrm{S}+\mathrm{L}$ & NGC1313 & SB & 0.0015 & 0.02 & $2 .^{i}$ & 8.44 & 0.61 & $0.65-0.60 \pm 0.03$ \\
\hline $\mathrm{C}$ & $\mathrm{S}+\mathrm{L}$ & NGC1510 & BCG & 0.003 & 0.00 & 5.1 & 8.37 & 0.08 & $0.09-1.72 \pm 0.02$ \\
\hline $\mathrm{K}$ & $\mathrm{S}+\mathrm{L}$ & NGC1569 & SB & 0.000 & 0.51 & 2.9 & 8.37 & 0.07 & $0.07-1.15 \pm 0.02$ \\
\hline $\mathrm{K}$ & $\mathrm{S}$ & NGC1614 & SB & 0.0159 & 0.05 & 3.8 & 8.84 & 0.93 & $1.00-0.65 \pm 0.12$ \\
\hline $\mathrm{C}$ & $\mathrm{S}+\mathrm{L}$ & NGC1672 & $\mathrm{SB}+\mathrm{Sy}$ & 0.0039 & 0.00 & $2 .^{i}$ & 9.14 & 0.60 & $0.64-0.01 \pm 0.02$ \\
\hline $\mathrm{C}$ & $\mathrm{S}+\mathrm{L}$ & NGC1705 & BCG & 0.0023 & 0.04 & 3.0 & 8.47 & 0.00 & $0.00-2.46 \pm 0.01$ \\
\hline $\mathrm{C}$ & $\mathrm{S}$ & NGC1800 & SB & 0.0024 & 0.00 & $2 .^{i}$ & 8.36 & 0.07 & $0.07-1.65 \pm 0.11$ \\
\hline $\mathrm{C}$ & $\mathrm{S}+\mathrm{L}$ & NGC3049 & SB & 0.005 & 0.01 & 0.0 & 8.90 & 0.31 & $0.33-1.11 \pm 0.03$ \\
\hline $\mathrm{C}$ & $\mathrm{S}+\mathrm{L}$ & NGC3125 & BCG & 0.004 & 0.07 & 1.0 & 8.36 & 0.13 & $0.14-1.53 \pm 0.03$ \\
\hline $\mathrm{C}$ & S & NGC3256 & SB & 0.0094 & 0.13 & 2.0 & 8.82 & 0.60 & $0.64+0.32 \pm 0.08$ \\
\hline $\mathrm{C}$ & $\mathrm{S}+\mathrm{L}$ & $1050+04$ & BCG & 0.021 & 0.03 & $2 .^{i}$ & 8.57 & 0.55 & $0.59-1.05 \pm 0.03$ \\
\hline $\mathrm{K}$ & $\mathrm{S}+\mathrm{L}$ & NGC4194 & BCG & 0.0081 & 0.00 & 1.5 & 8.81 & 0.80 & $0.86-0.25 \pm 0.03$ \\
\hline $\mathrm{C}$ & $\mathrm{S}+\mathrm{L}$ & NGC4385 & SB & 0.007 & 0.02 & 0.0 & 8.65 & 0.58 & $0.62-1.13 \pm 0.03$ \\
\hline $\mathrm{K}$ & $\mathrm{S}$ & Mrk66 & BCG & 0.03 & 0.00 & 5.7 & 8.39 & 0.00 & $0.00-1.60 \pm 0.06$ \\
\hline $\mathrm{C}$ & $\mathrm{S}+\mathrm{L}$ & NGC5236 & SB & 0.002 & 0.03 & $2 i^{i}$ & 9.31 & 0.29 & $0.31-0.83 \pm 0.03$ \\
\hline $\mathrm{C}$ & $\mathrm{S}+\mathrm{L}$ & NGC5253 & SB & 0.0009 & 0.05 & 5.6 & 8.39 & 0.00 & $0.00-1.46 \pm 0.01$ \\
\hline K & $\mathrm{S}+\mathrm{L}$ & UGC9560 & BCG & 0.004 & 0.00 & 5.1 & 8.42 & 0.15 & $0.16-2.04 \pm 0.02$ \\
\hline $\mathrm{K}$ & $\mathrm{S}+\mathrm{L}$ & NGC5860 & SB & 0.017 & 0.01 & 4.0 & 9.24 & 0.69 & $0.74-0.98 \pm 0.05$ \\
\hline $\mathrm{K}$ & $\mathrm{S}$ & UGCA410 & BCG & 0.0022 & 0.01 & 6.2 & 8.46 & 0.12 & $0.13-1.15 \pm 0.12$ \\
\hline $\mathrm{K}$ & $\mathrm{S}+\mathrm{L}$ & NGC5996 & SB & 0.011 & 0.02 & 3.4 & 8.93 & 0.47 & $0.50-0.99 \pm 0.04$ \\
\hline $\mathrm{K}$ & $\mathrm{S}+\mathrm{L}$ & NGC6052 & SB & 0.015 & 0.03 & 1.9 & 8.61 & 0.21 & $0.22-0.87 \pm 0.02$ \\
\hline $\mathbf{K}$ & $\mathrm{S}$ & NGC6090 & SB & 0.0293 & 0.00 & 0.8 & 8.77 & 0.60 & $0.64-0.78 \pm 0.09$ \\
\hline K & $\mathrm{S}$ & NGC6217 & SB & 0.0046 & 0.04 & 2.4 & 8.98 & 0.53 & $0.57-0.61 \pm 0.06$ \\
\hline K & $\mathrm{S}$ & Mrk499 & BCG & 0.0258 & 0.04 & $2 .^{i}$ & 8.47 & 0.45 & $0.48-1.02 \pm 0.07$ \\
\hline $\mathrm{C}$ & $\mathrm{S}+\mathrm{L}$ & NGC6221 & $\mathrm{SB}+\mathrm{Sy}$ & 0.005 & 0.20 & $2 .^{i}$ & 8.94 & 0.63 & $0.67+0.41 \pm 0.10$ \\
\hline $\mathrm{C}$ & $\mathrm{S}+\mathrm{L}$ & Tol1924-416 & BCG & 0.010 & 0.07 & 0.0 & 8.32 & 0.02 & $0.02-2.08 \pm 0.02$ \\
\hline $\mathrm{C}$ & $\mathrm{S}$ & $1941-543$ & SB & 0.0188 & 0.04 & 3.0 & 8.45 & 0.16 & $0.17-1.38 \pm 0.11$ \\
\hline $\mathrm{K}$ & $\mathrm{S}+\mathrm{L}$ & NGC7250 & SB & 0.004 & 0.15 & 3.9 & 8.65 & 0.09 & $0.10-1.47 \pm 0.04$ \\
\hline $\mathrm{C}$ & $\mathrm{S}+\mathrm{L}$ & NGC7496 & $\mathrm{SB}+\mathrm{Sy}$ & 0.005 & 0.00 & 1.4 & 8.89 & 0.45 & $0.48-0.95 \pm 0.06$ \\
\hline $\mathrm{C}$ & $\mathrm{S}+\mathrm{L}$ & NGC7552 & SB & 0.005 & 0.00 & $2 .^{i}$ & 9.03 & 0.70 & $0.75+0.43 \pm 0.02$ \\
\hline K & $\mathrm{S}+\mathrm{L}$ & NGC7673 & SB & 0.010 & 0.04 & 2.1 & 8.48 & 0.42 & $0.45-1.48 \pm 0.02$ \\
\hline K & $\mathrm{S}+\mathrm{L}$ & NGC7714 & SB & 0.0093 & 0.04 & 1.3 & 8.71 & 0.39 & $0.42-1.23 \pm 0.02$ \\
\hline $\mathrm{K}$ & $\mathrm{S}$ & Mrk542 & SB & 0.0245 & 0.02 & $2 .^{i}$ & 8.87 & 0.31 & $0.33-1.32 \pm 0.14$ \\
\hline $\mathrm{C}$ & $\mathrm{S}+\mathrm{L}$ & NGC7793 & SB & 0.001 & 0.00 & 2.2 & 8.64 & 0.44 & $0.47-1.34 \pm 0.04$ \\
\hline
\end{tabular}

NOTES.-(a) $\mathrm{K}=\mathrm{KPNO}, \mathrm{C}=\mathrm{CTIO}$. (b) $\mathrm{S}+\mathrm{L}=$ both IUE short and long-wavelength spectra are available. $\mathrm{S}=$ only $I U E$ short-wavelength spectra are available. (c) $\mathrm{SB}=$ starburst galaxy, BGC = blue compact galaxy. (d) Redshift. (e) Foreground extinction from our Galaxy. (f) Oxygen abundance, defined as $\mathrm{O} / \mathrm{H}=12+\log (\mathrm{O} / \mathrm{H})$. (g) Intrinsic extinction of the galaxy: $E(B-V)_{i}=E(B$ $-V)($ total $)-E(B-V)_{G}$. (h) Difference between the optical depths of $\mathrm{H} \alpha$ and $\mathrm{H} \beta$, as defined in $\S 3.1$. (l) Power-law index of the UV continuum: the fitted range is $1250-2600 \AA$ if $\mathrm{S}+\mathrm{L}$ IUE spectra are available; it is $1250-1950 \AA$ if only S IUE spectra are available.

${ }^{\mathrm{i}}$ The average value $\mathrm{EW}_{\mathrm{abs}}=2 \AA$ has been assumed (McCall et al. 1985). 
with a $10^{\prime \prime}$ width was used and then a window $20^{\prime \prime}$ long was extracted, therefore matching closely the $I U E$ aperture. The CTIO spectra cover the wavelength range 3200-10000 $\AA$ with a resolution $\sim 8 \AA$ (for more details on the observations, see Storchi-Bergmann, Kinney, \& Challis 1994b).

The data were flat-fielded, wavelength calibrated, flux calibrated, and atmospheric extinction corrected using standard IRAF packages. The spectra obtained at KPNO have been additionally corrected for the extinction induced by the eruption from Mount Pinatubo (NOAO Newsletter, 1991 December). To show the matching of the IUE and optical apertures, an example of UV + optical spectrum is plotted in Figure 2, with no normalization between the UV and the optical region.

For emission lines of the Balmer series, the [O II] $\lambda 3727$, the [O III] $\lambda \lambda 4959,5007$, the [N II] $\lambda 6584$, and the [S II $] \lambda \lambda 6717$, 6731 were measured in order to determine the total dust extinction of the galaxy, the underlying stellar absorption of the Balmer lines, the electron temperature and density, and the oxygen abundance (see, for more details, Storchi-Bergmann, Calzetti, \& Kinney 1994a). The uncertainties on the line measurements range from $5 \%$ for the most intense lines (e.g., $\mathrm{H} \alpha$, [O III] $25007,[\mathrm{O}$ II $] \lambda 3727$ ) to $25 \%$ for the faintest ones (such as $[\mathrm{N} \mathrm{II}] \lambda 6584$, which is blended with $\mathrm{H} \alpha$ ). The uncertainty in the measurement of $\mathrm{H} \gamma$ goes from $10 \%$ in almost unreddened galaxies to $50 \%$ in reddened galaxies. In cases where the uncertainty would have been higher than $50 \%$, we have not used the measurement of $\mathrm{H} \gamma$ for our analysis.

To the purpose of correcting for dust extinction the observed emission lines, we have derived the total color excess $E(B-V)_{t}$ from the Balmer line ratio $\mathrm{H} \alpha / \mathrm{H} \beta$ and, when available, $\mathrm{H} \gamma / \mathrm{H} \beta$, adopting the theoretical Balmer line ratios given in Osterbrock (1989) and the Seaton values for the optical extinction law. Where $\mathrm{H} \gamma$ is detected (and has an uncertainty smaller than $50 \%$ ), the Balmer line ratios have been corrected simultaneously for $E(B-V)_{t}$ and for the underlying stellar absorption, following the method illustrated by McCall, Rybski, \& Shields (1985). For the spectra where $\mathrm{H} \gamma$ is undetected or highly uncertain, an average underlying stellar absorption of $2 \AA$ has been assumed (McCall et al. 1985). The typical uncertainty on $E(B-V)_{t}$ is about \pm 0.1 . The quantity $E(B-V)_{t}$ is the sum of the color excess intrinsic to the galaxy, $E(B-V)_{i}$, and the color

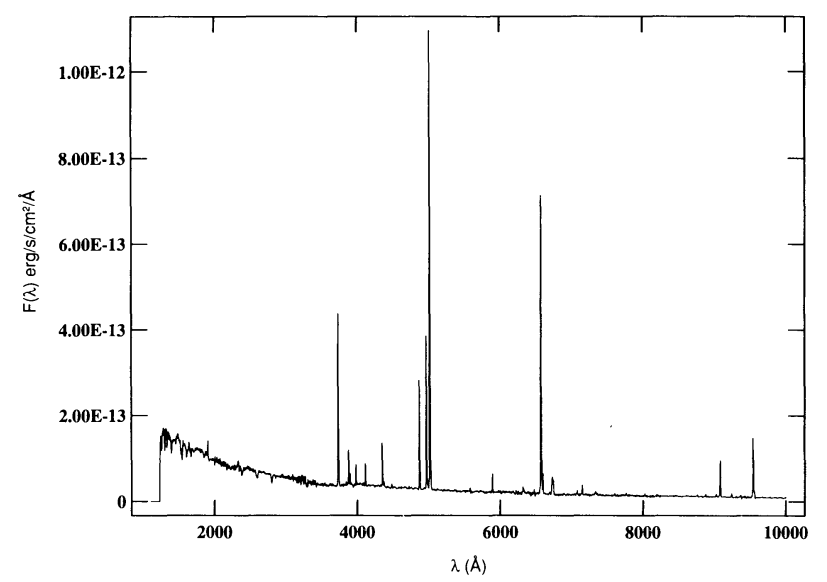

FIG. 2.-An example of UV and optical spectrum with no normalization between the two wavelength ranges. The galaxy is NGC 5253. The flux in ergs $\mathrm{cm}^{-2} \mathrm{~s}^{-1} \AA^{-1}$ is plotted as a function of the wavelength $\lambda$ in the range $1220-10000 \AA$. The joining point between the UV and optical is at $3200 \AA$. excess $E(B-V)_{G}$ given by the foreground extinction from our Galaxy. In order to derive $E(B-V)_{i}$, we have obtained $E(B-V)_{G}$ from the Galaxy extinction maps of Burstein \& Heiles (1982).

The method presented by McCall et al. (1985) for correcting the Balmer emission lines simultaneously for the total color excess $E(B-V)_{t}$ and for the underlying stellar absorption $\mathrm{EW}_{\mathrm{abs}}$ is based on two hypotheses: (a) the equivalent width of the stellar absorption underlying $\mathrm{H} \alpha$ is much smaller than the corresponding nebular emission line, $\mathrm{EW}_{\mathrm{abs}}(\mathrm{H} \alpha) \ll \mathrm{EW}_{\mathrm{em}}(\mathrm{H} \alpha)$; (b) the assumption $\mathrm{EW}_{\mathrm{abs}}(\mathrm{H} \alpha) \simeq \mathrm{EW}_{\mathrm{abs}}(\mathrm{H} \beta) \simeq \mathrm{EW}_{\mathrm{abs}}(\mathrm{H} \gamma)$ is valid. Hypothesis $(a)$ is always verified in our sample for values of the $\mathrm{H} \alpha$ stellar absorption $\mathrm{EW}_{\mathrm{abs}}(\mathrm{H} \alpha) \lesssim 5 \AA$; so hypothesis (b) does not constrain the $\mathrm{H} \alpha$ line, because the correction to the emission-line flux introduced by the stellar absorption is within a few percent. The assumption $\mathrm{EW}_{\mathrm{abs}}(\mathrm{H} \beta) \simeq \mathrm{EW}_{\mathrm{abs}}(\mathrm{H} \gamma)$ is valid within $3 \%$ for blue stellar populations (McCall et al. 1985). For red populations, the difference between the $\mathrm{H} \gamma$ and the $\mathrm{H} \beta$ stellar absorptions can be as large as $30 \%$ (Bruzual \& Charlot 1993), yielding to a variation in the color excess $\Delta E(B$ $-V)_{t} \sim \pm 0.05$, which is within our measurement uncertainties.

As an additional test to study the influence of the Balmer line absorptions of a red stellar population on the line emission fluxes, we have compared, where possible, the optical spectra of our galaxies to Bica's (1988) galaxy templates, by matching the stellar absorption lines not contaminated by emission. We have measured the $\mathrm{EW}_{\mathrm{abs}}(\mathrm{H} \beta)$ and $\mathrm{EW}_{\mathrm{abs}}(\mathrm{H} \alpha)$ of the templates and used these values to rederive $E(B-V)_{t}$. As before, we find that the typical variation is $\Delta E(B-V)_{t} \sim \pm 0.05$, which is within our uncertainties, except for IC 1586 and NGC 1313, where $\Delta E(B-V)_{t} \simeq-0.2$. However, for sake of homogeneity and because of the uncertain identification of the underlying red stellar population, we prefer to adopt McCall et al.'s method for all our galaxies. In addition, as can be seen from Table 1 and Figure $5 a$, our conclusions are not affected by a change as small as -0.2 in the color excess of IC 1586 and NGC 1313.

After correcting the optical emission lines for the dust extinction by using a Seaton (1979) law, we have determined the electron temperature and density, and the oxygen abundance of our galaxies. The electron density has been determined from the $[\mathrm{S} \mathrm{II}] \lambda \lambda 6717 / 6731$ line intensity ratio and the electron temperature $T[\mathrm{O}$ III $]$ from the calibration diagrams of Pagel et al. (1979) and also, in the temperature range 7000 $K<T[\mathrm{O} \mathrm{III}]<9000 \mathrm{~K}$, of Dopita \& Evans (1986). The oxygen abundance has been determined assuming an electron temperature for $[\mathrm{O} \mathrm{II}]$ given by the relation $T[\mathrm{O}$ II $]=0.7 T[\mathrm{O} \mathrm{III}]+3000$ (Campbell, Terlevich, \& Melnick 1986) and using the photoionization relations by McCall (1984). These formulae provide the dependence of the emission coefficients not only on the temperature but also on the electron density, thus accounting for the contribution of collisional deexcitation to the ion abundances, which becomes important in the case of low ionization species, such as $\mathrm{O}^{+}$, and for electron densities higher than about $300 \mathrm{~cm}^{-3}$ (13 of 39 galaxies in our sample have electron density higher than this limit). The values of the oxygen abundance obtained with the described technique have been compared with the abundances derived from the calibration diagrams of McGaugh (1991), and the difference between the two sets of values is within 0.1 dex.

The adoption of a Seaton law to deredden the metallic emission lines does not introduce a bias in the measured metal- 
licities. As we will see later, the larger variation in the value of the extinction we can expect is about a factor of 2 . This introduces a systematic variation of about 0.1 dex in the value of $\mathrm{O} / \mathrm{H}$, which is however within our uncertainties.

Keel et al. (1985) have shown that, in the case of highly reddened galaxies or $\mathrm{H}$ II regions, the dereddened value of the [O II] $\lambda 3727$ line flux can be unreasonably large when compared with expectations from model $\mathrm{H}$ II regions of comparable $[\mathrm{O} \mathrm{III}] / \mathrm{H} \beta$ values. They interpret this finding as evidence for ionization and dust stratification on similar scales: if the dust is more concentrated towards the center of the emitting gas where the ionization is higher, adopting the same color excess for [O $\mathrm{II}]$ and [O III] leads to an overcorrection of the [O II] flux. The main effect in our case would be an underestimation of the galaxy oxygen abundance. In order to investigate such a possibility, we have plotted the dereddened ratio of $[\mathrm{O} \mathrm{III}]$ to $[\mathrm{O} \mathrm{II}]$ against the dereddened ratio $([\mathrm{O} \mathrm{III}]+[\mathrm{O} \mathrm{II}]) / \mathrm{H} \beta$ in Figure 3 . This plot can be compared with the analogous plot by McCall et al. (1985) of $99 \mathrm{H} \mathrm{II}$ regions and with the theoretical diagram by McGaugh (1991) for models of photoionized nebulae. There is not much evidence for overcorrection of the [O II] emission-line flux in our sample, except probably for one galaxy, NGC 1313, (marked by two arrows in Fig. 3), and for two borderline cases, IC 1586 and NGC 7552 (each marked by an arrow in Fig. 3). NGC 1313 and IC 1586 are the same two galaxies for which the color excess $E(B-V)_{t}$ has been probably overestimated (see above). In order to understand how ionization and dust stratification can affect the value of the oxygen abundance in NGC 1313, IC 1586, and NGC 7552, we assume that the appropriate color excess for [O III] is the one given by the Balmer line ratio and adopt as a color excess for the [O II] emission line one-half of the corresponding [O III] color excess. The change in the oxygen abundance we obtain is $\Delta \mathrm{O} / \mathrm{H} \simeq 0.1$, which is within our measurement uncertainties.

The range of oxygen abundances covered by our sample is $8.3 \leq 12 .+\log (\mathrm{O} / \mathrm{H}) \leq 9.3$. In this paper we use the value of the oxygen abundance to divide the galaxies into two groups: the ones with metallicity close to the LMC value (LMC-like)

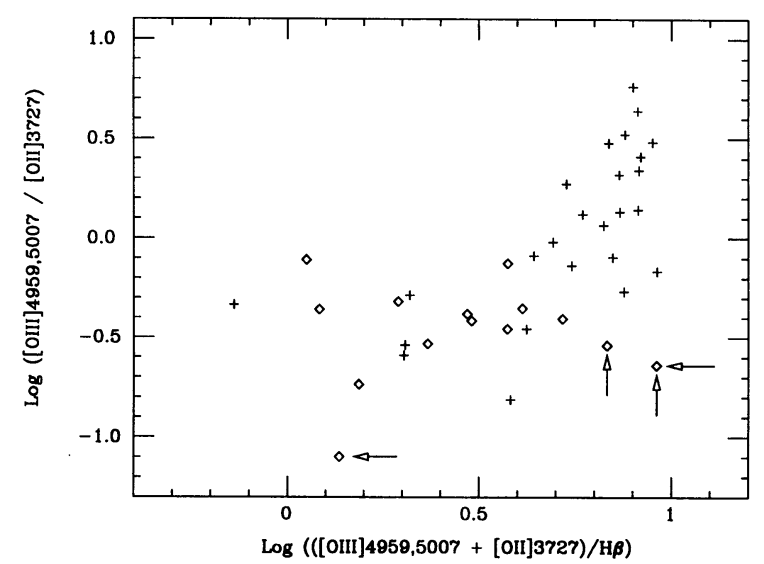

Fig. 3.-The dereddened ratio [O III] $\lambda \lambda 4959,5007 /[\mathrm{O} \mathrm{II}] \lambda 3727$ is shown as a function of the dereddened metallicity indicator $([\mathrm{O} \mathrm{III}]+[\mathrm{O} \mathrm{II}]) / \mathrm{H} \beta$ in a $\log -\log$ plot. The vertical crosses mark the galaxies with $E(B-V)_{i}<0.5$; the diamonds mark the galaxies with $E(B-V)_{i} \geq 0.5$. One data point, NGC 1313 (marked by two arrows) probably deviates from the typical distribution of $\mathrm{H}$ II regions in this diagram, due to reddening overcorrection of the $[\mathrm{O} \mathrm{II}]$ line. Two data points, IC 1586 and NGC 7552 (each marked by one arrow) are borderline cases. and the ones with metallicity close to the Milky Way value (MW-like). The separation point between the two groups is chosen to be the average point between the metallicity of LMC and of the Milky Way, i.e., $\mathrm{O} / \mathrm{H}=8.63$. Therefore, we do not need a precise determination of the oxygen abundance of the galaxies in our sample, although the method we have summarized above gives uncertainties of the order of $0.15 \mathrm{dex}$ (cfr. Storchi-Bergmann et al. 1994a).

Table 1 lists the ground-based observatory where the optical spectra were obtained, the available IUE spectra, the galaxy name, the galaxy type, the redshift, the foreground extinction from the Milky Way in terms of the color excess $E(B-V)_{G}$, the equivalent width of the stellar absorption underlying the Balmer lines $\left(\mathrm{EW}_{\mathrm{abs}}\right)$, the oxygen abundance, the color excess $E(B-V)_{i}$ intrinsic to the galaxy, the Balmer optical depth $\tau_{B}^{l}$ (see $\S 3.1$ ), and the power-law index $\beta$ of the UV continuum ( $\beta$ will be discussed below). The objects in Table 1 are listed in order of right ascension.

\section{THE EFFECT OF DUST EXTINCTION ON THE UV CONTINUUM}

\subsection{The Balmer Optical Depth}

The Balmer line ratio $\mathrm{H} \alpha / \mathrm{H} \beta$ allows us to characterize the dust extinction in the regions where the nebular lines are produced. In this paper, we will compare this dust indicator with properties of the UV spectral continuum to derive the characteristics of the dust extinction in the UV wavelength range.

In the previous section, we have derived for the galaxies in our sample the intrinsic color excess $E(B-V)_{i}$ (see also Table 1) from the Balmer line ratio. However, the derivation of the color excess is based on the assumption that the values of the extinction law at the level of the Balmer lines are known. This assumption is not relevant in $\S 5$, where we compare our data with a model for the dust configuration based on the MW and LMC extinction laws, but can be misleading in $\S 6$, where we derive an extinction curve directly from the data. Therefore, for sake of uniformity in notation, we introduce as a dust indicator the difference $\tau_{B}^{l}$ in optical depth between emission lines $\mathrm{H} \alpha$ and $\mathrm{H} \beta$.

For the simple case of a uniform layer of dust between the source of intensity $I_{\lambda}^{0}$ and the observer, the optical depth $\tau(\lambda)$ is defined as

$$
I_{\lambda}=I_{\lambda}^{0} e^{-\tau(\lambda)},
$$

where $I_{\lambda}$ is the observed intensity, and all the quantities depend on the wavelength $\lambda$. The difference in optical depth between the nebular emission lines $\mathrm{H} \alpha$ and $\mathrm{H} \beta$, that we will call from now on Balmer optical depth, is given by

$$
\tau_{B}^{l}=\tau_{\beta}-\tau_{\alpha}=\ln \left(\frac{\mathrm{H} \alpha / \mathrm{H} \beta}{2.88}\right),
$$

where the superscript $l$ indicates that $\tau_{B}$ has been obtained from emission lines, and 2.88 is the theoretical value expected for the unreddened ratio $\mathrm{H} \alpha / \mathrm{H} \beta$ (Osterbrock 1989). Clearly, the Balmer optical depth does not depend on any assumption on the extinction law. $\tau_{B}^{l}$ is related to the color excess $E(B-V)_{i}$ calculated in $\S 2$ through

$$
E(B-V)_{i}=\frac{1.086}{k(\mathrm{H} \beta)-k(\mathrm{H} \alpha)} \tau_{B}^{l} \simeq 0.935 \tau_{B}^{l},
$$

where $k(\lambda)$ is the MW or LMC extinction law as defined in 
Figure 1 and the value $k(\mathrm{H} \beta)-k(\mathrm{H} \alpha)$ is obtained from Seaton (1979).

\subsection{The UV versus Optical Dust Extinction}

In the optical wavelength range, the Balmer line ratios, and therefore the Balmer optical depth $\tau_{B}^{l}$, provide a measurement of the dust content (down to optical depth $\tau=1$ ) in the galaxy regions where the burst of star formation is located; in fact, the line intensities are not sensitive to the parameters of the ionized gas, such as electron temperature or density (Osterbrock 1989).

In the UV range covered by IUE (1200-3200 $\AA)$ no set of lines comparable to the Balmer lines exists for starburst galaxies, and the dust manifests itself in the depression of the intrinsic continuum. We can, in principle, relate the shape of the observed UV continuum to the dust extinction. Let us consider two extreme situations: an instantaneous burst of star formation and a region of constant star formation. In the case of the instantaneous burst, the stellar population generated by the burst is younger than $\sim 2 \times 10^{7} \mathrm{yr}$, since all the galaxies in our sample show nebular emission lines. In fact, as the stellar population ages beyond $\sim 2 \times 10^{7} \mathrm{yr}$, there are no surviving stars hot enough to ionize the surrounding gas. During the first $2 \times 10^{7} \mathrm{yr}$, the stars contributing to the flux in the range 1200$3200 \AA$ (mainly B stars) have had no time to evolve off the main sequence, so that the shape of the intrinsic spectrum in the UV wavelengths of interest has not changed within the uncertainties of the typical IUE spectrum (C. Leitherer 1993, private communication). In the case of a region of constant star formation, the continuous generation of new stars keeps the shape of the UV spectrum roughly constant (Bruzual \& Charlot 1993). The stellar populations generated in a region of continuous star formation and the stellar population generated by an instantaneous burst, and younger than $2 \times 10^{7} \mathrm{yr}$, show a difference in the shape of their UV spectra, in the range 1200$2600 \AA$, of about $13 \%$, still within our measurement uncertainties. In the hypothesis that the two cases of instantaneous burst and of constant star formation bracket the possible situations in our sample of starburst galaxies and that there are no differences in the IMF between different galaxies, all our galaxies are characterized by a single intrinsic shape for the UV spectrum and all the deviations from that shape can be attributed to dust extinction. We will see in $\S 3.3$ that we have to relax this simplifying hypothesis, although its main consequences remain valid.

In order to study the effects of dust extinction on the UV continuum, we have fitted the observed UV continuum [corrected for the foreground extinction $E(B-V)_{G}$ ] of the galaxies in our sample to a power law expressed as

$$
F(\lambda) \propto \lambda^{\beta},
$$

in the range $1250 \leq \lambda \leq 2600 \AA$, where $F(\lambda)$ is the observed flux in ergs $\mathrm{s}^{-1} \mathrm{~cm}^{-2} \AA^{-1}$ and $\beta$ is the power-law index. We have correlated $\beta$ with the Balmer optical depth $\tau_{B}^{l}$.

The UV spectra in our sample are characterized by the presence of many stellar and interstellar absorption features, which affect the fitting procedure and alter artificially the shape of the continuum. To avoid this problem, we have selected 10 windows in the range 1200-2600 $\AA$ (see Table 2), to be used for the fit of the continuum. the 10 windows have been designed to remove from the fitting procedure all the relevant absorption features and $2175 \AA$ dust feature. The dust feature has been removed for two reasons: the first is that the region around $2200 \AA$ is the noisiest region in the IUE cameras; the second is
TABLE 2

FiTTING WiNDOWS

\begin{tabular}{|c|c|}
\hline $\begin{array}{l}\text { Window } \\
\text { Number }\end{array}$ & $\begin{array}{l}\text { Wavelength Range } \\
(\AA)\end{array}$ \\
\hline 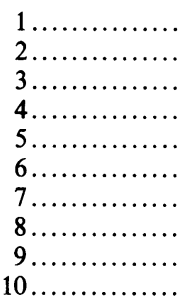 & $\begin{array}{l}1268-1284 \\
1309-1316 \\
1342-1371 \\
1407-1515 \\
1562-1583 \\
1677-1740 \\
1760-1833 \\
1866-1890 \\
1930-1950 \\
2400-2580\end{array}$ \\
\hline
\end{tabular}

that, if present, the $2175 \AA$ bump is the strongest deviation from the power-law approximation of the observed spectrum. However, the presence or absence of the $2175 \AA$ dust feature is the clearest indicator of a MW-type dust composition, and it will be discussed in $\S \S 5$ and 6 . We have not selected windows longward of $2600 \AA$ to avoid heavy contribution from stars of spectral type later than $F 2$. The fitting routine applies a linear least squares minimization to the data in the $\log F(\lambda)-\log (\lambda)$ plane. Figure 4 shows two examples of fits to the continuum for two galaxies of our sample, in two extreme cases of a spectrum with high signal-to-noise ratio and a spectrum with low
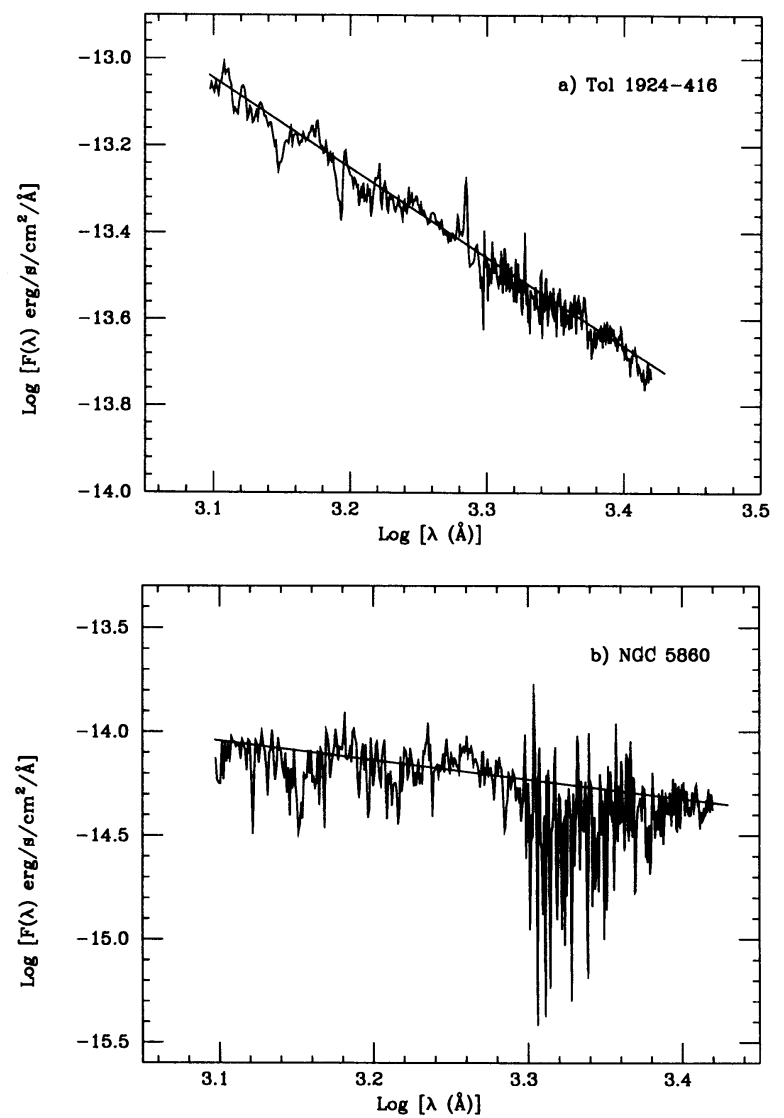

Fig. 4. $-(a)$ The spectrum $\log [F(\lambda)]$ vs. $\log (\lambda)$ and the fit of the continuum in the range 1200-2600 $\AA$ of Tol 1924-416, with an IUE spectrum of high signal-to-noise ratio. (b) The same as (a), but for NGC 5860, with an IUE spectrum of relatively low signal-to-noise ratio. 

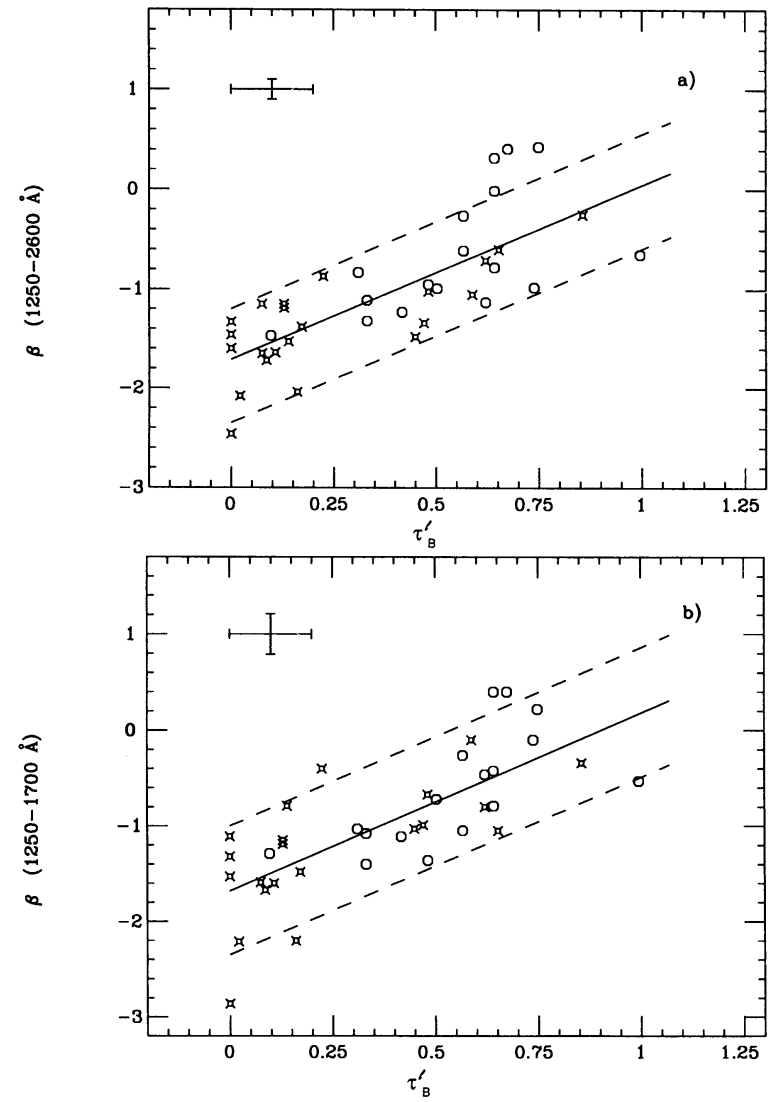

Fig. 5.-The UV power-law index $\beta$ is shown as a function of the Balmer optical depth $\tau_{B}^{l}$ (eq. [2]). The bluer galaxies have small values of $\beta$ (close to $\beta=-2$ ), and the redder galaxies have large values of $\beta$ (close to $\beta=0$ ). (a) The fitting routine to obtain $\beta$ has made use of the 10 windows listed in Table 2, so the wavelength range fitted is $1250-2600 \AA$. (b) $\beta$ has been obtained in the range 1250-1700 $\AA$. In both figures the $1 \sigma$ error bars are shown in the upper left-hand corner; the best linear fit is shown as a continuous line, and the upper and lower envelopes to the data are drawn as dashed lines. The asterisks indicate galaxies with LMC-like metallicity, and the circles indicate galaxies with MW-like metallicity.

signal-to-noise ratio. In both cases the fit to a power law is remarkably good.

Figure $5 a$ reports the power-law indexes $\beta$, obtained with the procedure described above, as a function of $\tau_{B}^{l}$ for the 39 galaxies of the sample. Different symbols distinguish LMC-like and MW-like galaxies; however, the two groups of galaxies do not show different trends in the plane $\beta-\tau_{B}^{l}$. We will discuss in $\S 3.5$ the meaning of the common trend manifested by LMClike and MW-like galaxies. Meanwhile, in our next considerations, we will assume that the two groups of galaxies are not physically distinct.

In Figure $5 a, \beta$ is clearly correlated with $\tau_{B}^{l}$ and the dashed lines in the figure mark the approximate envelope of the points. A Spearman nonparametric correlation test gives a coefficient $r_{s}=0.83$ and the points deviate $5.2 \sigma$ from the hypothesis of zero correlation. The envelope encloses almost all the points, suggesting that the relation between $\beta$ and $\tau_{B}^{l}$ might be linear. A linear least-squares fit gives

$$
\beta=(1.76 \pm 0.25) \tau_{B}^{l}-(1.71 \pm 0.12) .
$$

The best fit equation (4) is shown as a continuous line in Figure $5 a$. We do not include the error bars of the data points in our fit, because the main source of uncertainty of the fit parameters is the intrinsic spread of the data.

From equation (4), the bluest spectrum is given by the value of $\beta$ at zero extinction $\left(\equiv \tau_{B}^{l}=0\right), \beta_{0}=-1.71 \pm 0.12$, which is very close to the theoretical value $\sim-2$ obtained from spectral synthesis models (e.g., Bruzual \& Charlot 1993). This suggsts that the fit given by equation (4) is reasonable.

\subsection{How Variations in the Stellar Population Affect $\beta$}

Before interpreting the correlation between $\beta$ and $\tau_{B}^{l}$ in terms of dust extinction of the UV stellar continuum and turning to the comparison of the data with models for the geometrical distribution of dust, we have to exclude the possibility that the correlation is produced, partially or entirely, by changes in the quantities linked to the burst of star formation or to the star formation history. We must indeed answer two questions: (1) Are there other quantities which correlate with both $\beta$ and the dust content in the galaxy and, therefore, contribute to or produce entirely the correlation between $\beta$ and $\tau_{B}^{l}$ and (2) what is the origin of the spread in $\beta$ for constant values of $\tau_{B}^{l}$ ?

The oxygen abundance $\mathrm{O} / \mathrm{H}$ correlates mildly with the Balmer optical depth $\tau_{B}^{l}$ (see Table 1 and Storchi-Bergmann et al. 1994a): in fact, metal-rich environments are expected to be dustier than metal-poor environments. As a consequence of this correlation and of equation (4), $\beta$ also correlates with $\mathrm{O} / \mathrm{H}$. The correlation between $\beta$ and $\mathrm{O} / \mathrm{H}$ (shown in Figure 6) is weaker than the correlation between $\beta$ and $\tau_{B}^{l}$, but still present. A Spearman nonparametric correlation test gives a correlation coefficient $r_{s}=0.63$ and the points deviate $4 \sigma$ from the hypothesis of zero correlation.

A correlation between $\beta$ and metallicity can be caused not only by the increase in the dust content in more metal-rich galaxies, but also by the variation of quantities which are intrisic to the star formation history of the galaxy. In other words, we want to ask if metal-poor galaxies (which are mostly BCG in our sample) can be expected to have an intrinsically bluer spectrum than metal-rich galaxies (which are mostly starbursts in our sample) and how important the difference is.

If the slope and/or the upper mass limit of the stellar initial mass function (IMF) depend on the metallicity (see, e.g., Shields \& Tinsley 1976; Terlevich \& Melnick 1981), the intrinsic UV spectrum of the burst of star formation can, in principle,

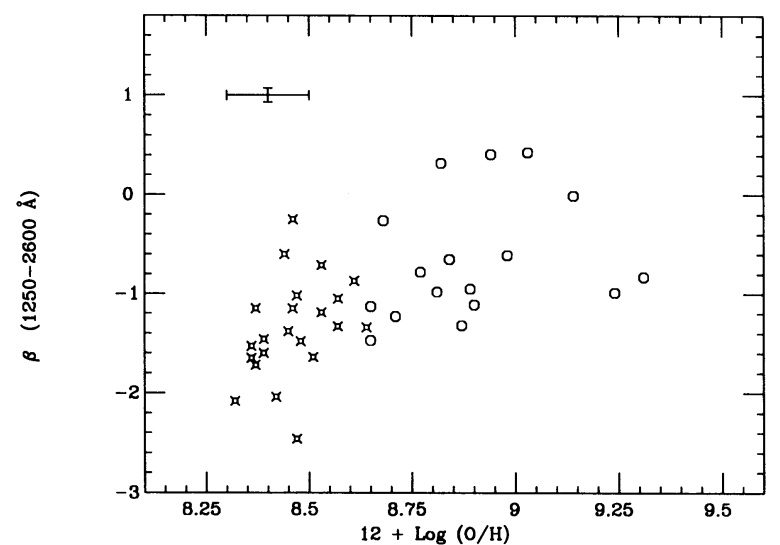

Fig. 6.-The UV power-law index $\beta$ is shown as a function of the oxygen abundance $\mathrm{O} / \mathrm{H}$. The typical $1 \sigma$ error bar is shown in the upper left-hand corner of the figure. $\beta$ is correlated with $\mathrm{O} / \mathrm{H}$, although the correlation is weaker than in the case of $\beta$ vs. $\tau_{B}^{l}$ (see Fig. $4 a$ and the text). The symbols for the data points are as in Fig. 5. 
be affected resulting in a correlation between $\beta$ and $\mathrm{O} / \mathrm{H}$. In addition, more metal-rich and, probably, older starburst galaxies may have residual stellar populations from previous bursts of star formation (e.g., A stars), which redden the UV spectrum, thus mimicking a correlation between $\beta$ and $\mathrm{O} / \mathrm{H}$.

We have to investigate the impact of these parameters, changes in the IMF and presence of populations of A stars, on the correlation between $\beta$ and $\mathrm{O} / \mathrm{H}$, and, therefore, on the correlation between $\beta$ and $\tau_{B}^{l}$.

An evolution of the IMF produced only by a change of its upper mass limit, $100 M_{\odot}>M_{\text {up }}>25 M_{\odot}$ (cf. Shields \& Tinsley 1976), affects the shape of the spectrum longward of $1200 \AA$ by less than a few percent in our range of metallicity, $8.3 \lesssim \mathrm{O} / \mathrm{H} \lesssim 9.3$ (C. Leitherer 1993 , private communication). A change in the IMF slope would introduce a slightly higher deviation in the shape of the spectrum. However, the pattern of the evolution of the IMF with the metallicity is still a controversial topic (see, e.g., Vilchez \& Pagel 1988; Vilchez et al. 1988; Campbell 1988; Peña, Ruiz, \& Maza 1991, and, for a review, Kennicutt 1991). The change in the stellar evolutionary tracks due to variations in the metal content of the stars affects the UV spectrum by less than $10 \%$, which is within our measurement uncertainties. Thus we can conclude that the correlation between the IMF and the metallicity has a small effect on the correlation between $\beta$ and $\mathrm{O} / \mathrm{H}$, and, thus, on the correlation between $\beta$ and $\tau_{B}^{l}$.

The metal enrichment of galaxies is the by-product of consecutive bursts of star formation, or of a constant star formation rate. If the metal-rich galaxies in our sample have undergone various bursts of star formation relatively close in time, a substantial population of A stars could be still surviving, because of the long life of $A$ stars $\left(\gtrsim 10^{9} \mathrm{yr}\right)$ in comparison to B stars $\left(\sim 10^{8} \mathrm{yr}\right)$. On the other hand, the metal-poor galaxies in our sample could be experiencing the first, or one of the first, bursts of star formation, so that A stars would not constitute a relevant contributor to the UV radiation. This effect may mimic a trend of $\beta$ versus $\mathrm{O} / \mathrm{H}$ (or $\tau_{B}^{l}$ ), since A stars, which have nonzero flux only longward of $1600 \AA$, redden the UV spectrum $(\beta \rightarrow 0)$. Therefore, it is important to test if the increase in value of $\beta$ for increasing $\mathrm{O} / \mathrm{H}$ is due only to dust extinction, or if it is also due to the contribution from previous generations of stars in the older (metal-rich and dustier) galaxies.

In order to test the importance of A stars in the UV flux, we have repeated the continuum fitting procedure described above using windows 1-6 (range 1250-1700 $\AA$; see Table 2). Stars of spectral type later than A2 do not contribute to the flux at wavelengths shorter than $1700 \AA$. The resulting $\beta$ for the 39 galaxies are shown in Figure $5 b$; the $1 \sigma$ error bars in this case are about 3 times larger than in the previous analysis when all 10 windows were used. The correlation between $\beta$ and $\tau_{B}^{l}$ is still present (see Fig. 5b). A Spearman nonparametric correlation test gives a coefficient $r_{s}=0.80$ and the points deviate $4.9 \sigma$ from the null hypothesis. A linear least-squares fitting of the data points yields

$$
\beta=(1.86 \pm 0.28) \tau_{B}^{l}-(1.68 \pm 0.13) .
$$

The difference between equation (5) and equation (4) is negligible within the uncertainties. Hence we can conclude that the correlation between $\beta$ and $\tau_{B}^{l}$ is not affected by long-living stellar populations, and only the stars which are actively produced in the present burst (or bursts) of star formation contribute to the overall value of $\beta$.
In the following, we will use the data from Figure $5 a$ instead of Figure $5 b$, since those in Figure $5 a$ have a smaller uncertainty.

As discussed in $\S 3.2$, we expect that the UV spectra of young bursts of star formation and of regions of constant star formation rate have similar intrinsic shapes in the range 1250-2600 $\AA$. What is, then, the cause of the spread in $\beta$ for constant values of $\tau_{B}^{l}$ ? From Figure $5 a, \beta_{0}$ assumes values in the range -1.2 to -2.4 , a spread much higher than the formal $1 \sigma$ error bar obtained from the fitting of the continuum equation (4). The conclusion is that the spread is intrinsic and depends on deviations from the simple picture described in $\S 3.2$.

The intrinsic spread in $\beta$ can be due to the contribution to the UV spectrum from more than one burst of star formation at different stages of evolution. In fact, the IUE aperture is large enough to encompass a major fraction of the extension of the galaxies in the sample and, in principle, more than one bursting region. While some of these regions may still actively form stars, some others may have "exhausted their fuel" and their hot stars started to evolve off the main sequence. As a result, the overall UV spectrum reddens toward higher values of $\beta$. In addition, $O B$ associations tend to drift away from the parent molecular cloud (Mezger \& Smith 1977; Israel 1978; Garmany, Conti, \& Chiosi 1982; de Jong \& Brink 1986) on a time scale of about $3 \times 10^{6} \mathrm{yr}$. More evolved OB associations are then, embedded in less opaque regions and contribute, proportionally, a larger amount to the UV flux than younger, but more extincted, $\mathrm{OB}$ associations.

As an independent possibility, the spread in $\beta$ can be linked to variations in the relative spatial distribution between the dust and the ionized regions. In fact, the same amount of dust gives different extinctions for different geometrical distributions of the dust itself (see $\S 5$ below). However, it is not clear if this explanation is valid also in the limit $\tau_{B}^{l} \rightarrow 0$; on the one hand, the most metal-poor galaxies in our sample are onefourth solar; therefore, they should contain at least some dust despite the fact that, from the Balmer line ratio, the dust content is formally zero $\left(\tau_{B}^{l}=0\right)$; on the other hand, metalpoor galaxies are known to be less efficient at producing dust than metal-rich galaxies, and they might be relatively transparent systems (Hunter et al. 1989).

Although $\beta$ has an intrinsic spread for constant values of $\tau_{B}^{l}$, in Figure $5 a$ we can see that the intrinsic spread in $\beta$ is approximately constant along the sequence of $\tau_{B}^{l}$, and in what follows we will deal with the correlation $\beta-\tau_{B}^{l}$ as a monoparametric correlation, adopting as an index for the intrinsic (unreddened) spectrum the average value of $\beta_{0}=-1.71$ (see eq. [4]).

\subsection{Selection Effects in the Data}

There are two possible selection effects that, if present, would affect the results: (1) If two galaxies have the same UV integrated flux, the one having the bluer spectrum is detectable in the far-UV with a higher signal-to-noise ratio than a galaxy having a redder spectrum; (2) The galaxies in our sample are all observed with a fixed aperture in the UV and in the optical wavelengths; if there is a correlation between one of the parameters we are analyzing, for instance $\tau_{B}^{l}$, and the size of the sampled region within each galaxy, this can bias the correlation between $\beta$ and $\tau_{B}^{l}$. A correlation between $\tau_{B}^{l}$ and the size of the sampled region is possible, since the color excess displays a dependence on the position in the galaxy (see, e.g., Roy, Belley, \& Walsh 1989).

The first point can introduce a bias in the sample because 
spectra with very low signal-to-noise ratios have been excluded from the atlas of UV spectra (see Kinney et al. 1993). However, a bias in this sense would manifest itself as a decrease of the intrinsic spread of $\beta$ for increasing $\tau_{B}^{l}$. Figures $5 a$ and $5 b$ show that this is not the case: the intrinsic spread in $\beta$ remains roughly constant along the $\tau_{B}^{l}$ axis.

In order to understand if $\tau_{B}^{l}$ is correlated with the size of the sampled region in the galaxies, we have plotted the mean diameters of the galaxies, expressed as $D=\left[\left(d_{\min }^{2}+d_{\max }^{2}\right) / 2\right]^{1 / 2}$, where $d_{\min }$ and $d_{\max }$ are the minor and major angular axes, as a function of $\tau_{B}^{l}$ (see Fig. 7). A Spearman nonparametric correlation test gives a correlation coefficient $r_{s}=0.19$ and the points deviate $1.2 \sigma$ from the hypothesis of zero correlation, implying that they are compatible with the null hypothesis within $26 \%$. If we remove from the sample the four largest galaxies, the compatibility with the null hypothesis increases to $67 \%$. Therefore, we conclude that the observational technique has not introduced a bias in the parameter space we are analyzing.

\subsection{Metallicity and Extinction Law}

As mentioned in the Introduction, one of the hypotheses used to explain the difference in the far-UV between the MW, LMC, and SMC extinction laws is the difference in metallicity between the three galaxies (see, e.g., Lequeux 1988).

The three extinction laws are differentiated by an overall slope which becomes steeper in the far-UV (below $2600 \AA$ ) going from the MW to the $\mathrm{LMC}$, to the SMC, and by a weakening of the $2175 \AA$ dust feature. In our fitting procedure to obtain $\beta$ we have not included the region around $2175 \AA$, so $\beta$ is sensitive only to variations in the overall slope of the UV extinction law. For the optical wavelengths, there are no large differences between the three laws, so $\tau_{B}^{l}$ is a measurement of the extinction in all three cases.

In our sample, the galaxies are divided between those with LMC-like metallicity and those with MW-like metallicity (see Fig. 5a). The expectation, based on the known extinction laws, is that, for the same value of $\tau_{B}^{l}$, galaxies with LMC-like metallicity are on average redder, i.e., have an higher value of $\beta$, than galaxies with MW-like metallicity. We can verify this expectation looking at Figure $5 a$ (or Fig. $5 b$ ). There are seven LMC-

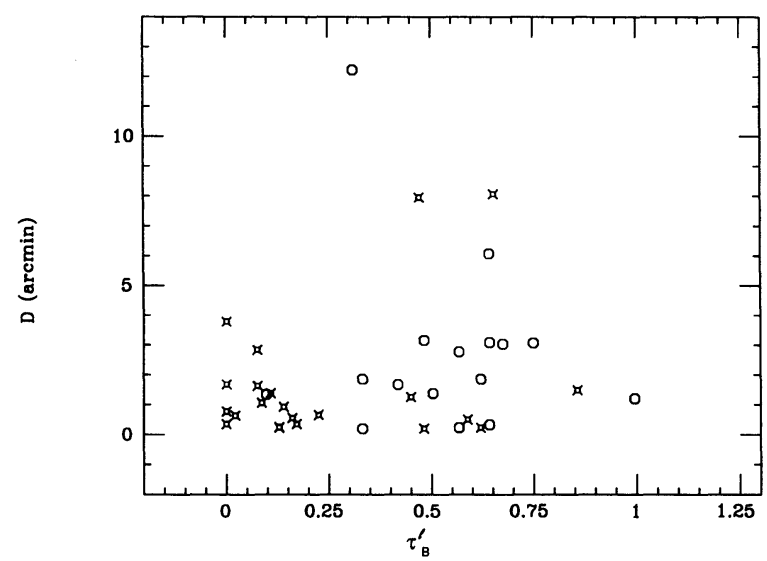

FIG. 7.- The mean angular diameters $D$ of the galaxies, expressed in arcminutes, are shown as a function of $\tau_{B}^{l}$. There is no obvious correlation between the two quantities. $\tau_{B}^{l}$ is obtained from the emission lines observed in the $\sim 10^{\prime \prime} \times 20^{\prime \prime}$ optical aperture. The lack of correlation between $D$ and $\tau_{B}^{l}$ means that the fixed aperture does not introduce a bias in the observed quantities, although the angular sizes of the observed galaxies, and therefore the sampled regions, change by large amounts. like galaxies with $0.43 \lesssim \tau_{B}^{l} \lesssim 0.86$. According to the expectation, these galaxies should occupy the region of high values of $\beta$ in the plot, higher, at least, than the region occupied by MW-like galaxies with analogous values of $\tau_{B}^{l}$. This is not the case.

In Figure $5 a$ (but also in Fig. $5 b$ ), there is no clear dichotomy between the $\beta$ - versus $-\tau_{B}^{l}$ trend of the LMC-like and of the MW-like galaxies: they can be described by a unique relation; the data are consistent with a unique extinction law independent of metallicity. A possible interpretation of this fact is that a higher metal content in the galaxy may increase the number of dust grains, without affecting the composition or the sizes' distribution (cf. Calzetti et al. 1993).

One caveat to this picture is the possibility that the absence of dichotomy in the behavior of LMC-like and MW-like galaxies is partially due to effects of a complex distribution for the dust, which may remove part of the differences between the two sets of galaxies.

\subsection{The Origin of the Nebular Lines and of the FUV Continuum}

Systematic studies of $\mathrm{H}$ II regions have shown that the OB associations tend to drift away from or to blow out the parent molecular cloud, reaching intercloud regions characterized by a very low density medium (e.g., Garmany et al. 1982; Leisawitz \& Hauser 1988). The typical traveling time is in the range 1-3 $\times 10^{6}$ yr (de Jong \& Brink 1986; Leisawitz \& Hauser 1988). Observational evidence for asymmetric distributions of molecular clouds and OB associations has been provided by Israel (1978).

After $3 \times 10^{6} \mathrm{yr}$, stars of spectral type earlier than 05 have evolved and the total number of Lyman-continuum photons produced by the $\mathrm{OB}$ association begins to decrease (Leitherer 1990). If we assume that the process of separation between the molecular cloud and the $\mathrm{OB}$ association progressively leads the OB association toward regions of lower extinction, an extreme scenario is that the UV continuum $(\lambda>1200 \AA)$ produced by the $\mathrm{OB}$ association is less extincted when there are fewer ionizing photons available to generate the nebular lines.

This process is not important if we are dealing with a single bursting region, with a one-age population. However, in the case of a burst of star formation lasting a time interval $t \gtrsim 10^{7}$ $\mathrm{yr}$, and for the large $I U E$ aperture which may encompass various bursting regions, it is difficult to understand if the main contribution to the UV continuum is given by the same stellar population which generates the Balmer lines. A realistic scenario for a starburst galaxy is that the UV continuum is partially or mostly produced by relatively evolved and little extincted $O B$ associations, while the nebular lines are mostly produced by hot and young, but more heavily extincted, OB associations. Such a scenario can explain the intrinsic spread in $\beta$ for constant values of $\tau_{B}^{l}$, as mentioned in $\S 3.3$, because the relative weight of the various components is not constant. However, it represents an objective difficulty in comparing the $\beta$ versus $\tau_{B}^{l}$ relation with the models for the dust distribution, since for these models the implicit assumption of a common origin for Balmer lines and UV continuum is made. In $\$ 4$ and 5 we will adopt this simplifying assumption, but we will relax it in $\S 6$.

\section{THE MODELS}

The fact that the UV continuum of the galaxies in our sample can be represented by a monoparametric relation as a 
function of the optical extinction $\tau_{B}^{l}$ means that starburst galaxies share common properties for the dust extinction in the UV. The next step is to understand which scenario of dust distribution reproduces the observed characteristics.

\subsection{Description}

We discuss five models for the relative dust/gas distribution, following a scheme analogous to the one described by Caplan \& Deharveng (1986) in their study of the reddening properties of the LMC in the optical and radio wavelengths. The five models encompass different configurations of the relative distribution of ionized gas and dust, schematizing various physical situations inside a galaxy.

The five models can be described as follows (see Fig. 8):

1. Uniform dust screen. This is the classical configuration assumed when dereddening single stars and may be an oversimplification in the case of galaxies. We include this case for comparison with the other models. In this model the screen is physically distant from the cloud of ionized gas, so the effect of dust is to extinct the flux from the source, namely to remove photons from the line of sight through absorption and scattering by dust grains.

2. Clumpy dust screen. As in model 1, the dust is located in a screen distant from the cloud of gas. The difference from the previous case is that the dust is organized in clumps. This configuration can be considered more realistic than model 1.

3. Uniform scattering slab. The dust is located close to the source of radiation, implying that the emitting region subtends a large angle centered on the dust grain. In this case the action of the scattering by dust is not only to remove photons from the line of sight (contributing, with the absorption, to the extinction), but also to convey photons back into the line of sight. Scattering, then, provides an important positive contribution to the emerging radiation.

4. Clumpy scattering slab. As in model 3, but dust is clumped.

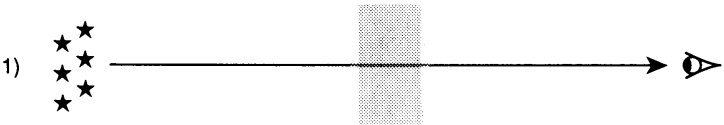

2)

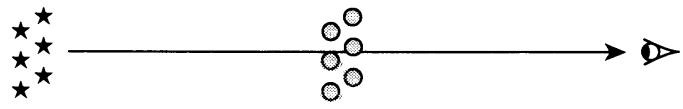

3)

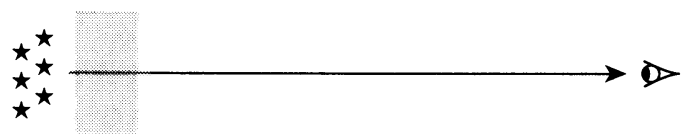

4)

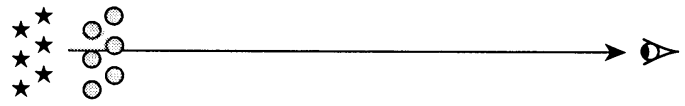

5)

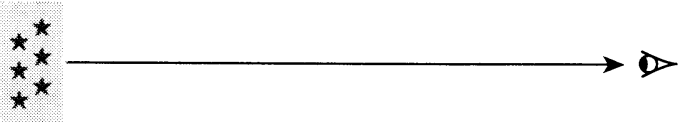

FIG. 8.-A schematic representation of the five configurations of dust/ ionized gas discussed in $\S 4$. From top to bottom, they are (1) the uniform dust screen; (2) the clumpy dust screen; (3) the uniform scattering slab; (4) the clumpy scattering slab; (5) the internal dust model.
5. Internal dust. Dust and ionized gas are uniformly mixed and the model schematizes the situation in which dust internal to the OB stellar association is present.

Our sample contains 22 galaxies with LMC-like metallicity and 17 galaxies with $\mathrm{MW}$-like metallicity. In order to account for all the possible parameters in five configurations, we will analyze the models using both the Milky Way and the LMC extinction laws as fitted by Seaton (1979) and Fitzpatrick (1986). While in the UV wavelength range there are noticeable differences between the MW and the LMC extinction laws (see Fig. 1), in the optical wavelength range, $\lambda \gtrsim 3000 \AA$, the difference between the two curves is negligible; therefore, optical parameters, such as $\tau_{B}^{l}$, can be determined indifferently with one curve or the other.

\subsection{Properties}

To clarify the notation we will use in the comparison between observations and models, we briefly recall the properties of the radiative transfer in a plane-parallel slab of dust and ionized gas (Sobolev 1983; see also Bruzual et al. 1988). The equation of radiative transfer for this case is

$$
\cos \theta \frac{\partial I_{\lambda}}{\partial z}=-\kappa_{\lambda} I_{\lambda}+\epsilon_{\lambda}+\kappa_{\lambda} \frac{\omega_{\lambda}}{4 \pi} \int I_{\lambda} \Phi(\cos \Theta) d \Omega,
$$

where $\theta$ is the angle between the direction perpendicular to the plane and the direction of the light beam, $I_{\lambda}$ is the radiation intensity, $\kappa_{\lambda}$ is the extinction coefficient of the dust (given by the sum of the absorption and the scattering coefficients), $\epsilon_{\lambda}$ is the emissivity of the sources embedded in the slab, $\omega_{\lambda}$ and $\Phi(\cos \Theta)$ are the albedo and the phase function of the dust grains, respectively, and $d \Omega=d \cos \theta d \phi$ is the element of solid angle as seen from the dust grain (Sobolev 1983). The albedo is defined as the ratio of the scattering to the extinction coefficients. The phase function depends on $\Theta$, which is the angle between the directions of the incident and scattered photon.

The first term on the right-hand side of equation (4) is the loss of intensity in the beam due to absorption and scattering of the incident radiation. The second term is the contribution to the emerging flux due to the sources in the slab. The third term accounts for the positive contribution to the intensity due to scattering of the light back into the beam. The scattering is described through the albedo (which is the probability of a photon to be scattered) and the phase function (which is the probability of a photon to be scattered in a certain direction). $\Phi(\cos \Theta)$ is usually described with a Henyey-Greenstein function by introducing the phase parameter $g_{\lambda}(\Theta)$, which is the average cosine of the angle of scattering (e.g., White 1979).

In equation (6) the dust emissivity has been neglected, since it becomes important in the infrared, while we are interested in the optical and UV wavelength regions.

We are interested in specifying equation (6) in detail for each of the five models described above, and relating it to the observed quantities $\beta$ and $\tau_{B}^{l}$.

1. The uniform dust screen is detached from the cloud of ionizing gas, which means $\epsilon_{\lambda}=0$, and the third term on the right-hand side of equation (6) is zero. The solution of equation (6) is, in this case, given by equation (1), where

$$
\tau(\lambda)=\kappa_{\lambda} z / \cos \theta
$$

is the extinction optical depth of the uniform slab. The optical depth is linked to the extinction in magnitudes $A(\lambda)$ through 
the relation (e.g., Mathis 1983):

$$
\tau(\lambda)=0.921 A(\lambda)=0.921 k(\lambda) E(B-V)_{i},
$$

where $E(B-V)_{i}$ is defined in $\S \S 2$ and 3.1. $\tau_{B}^{l}$ is obtained from the Balmer line ratio through equation (2).

Equation (8) is used to relate the MW and LMC extinction laws given in Figure 1 to the optical depth used in the text.

2 . In the clumped dust screen, the second and third terms on the right-hand side of equation (6) are still zero. To derive an analytic expression for the extinction of the emerging radiation, we can follow Natta \& Panagia (1984) and assume that in the clumped configuration all the clumps have the same optical depth $\tau_{c}(\lambda)$ and are Poissonian distributed, with an average of $\mathscr{N}$ clumps along the line of sight. In an inhomogeneous configuration the average optical depth $\tau_{m}(\lambda)$, defined as the total optical depth of the average number of clumps along the line of sight, and the effective optical depth $\tau_{\text {eff }}(\lambda)$, defined as $-\log \left(I / I^{0}\right)$ where $I$ is the emerging radiation and $I^{0}$ is the unextincted radiation, have different expressions (Natta \& Panagia 1984). In our model 2, we have:

$$
\begin{gathered}
\tau_{m}(\lambda)=\mathscr{N} \tau_{c}(\lambda) \\
\tau_{\text {eff }}(\lambda)=\mathscr{N}\left[1-e^{-\tau_{c}(\lambda)}\right] .
\end{gathered}
$$

$\tau_{m}(\lambda)$ is a measure of the amount of dust in front of the emitting region, while $\tau_{\text {eff }}(\lambda)$ gives a measure of the effects of the dust on the emerging radiation. In this case, the derivation of $\tau_{B}^{l}$ from equation (2) gives only an effective $\tau_{B}^{l}$, which is linked to the true optical depth of the Balmer lines via

$$
\tau_{B}^{l}=\mathscr{N}\left[e^{-\tau_{c}(\mathbf{H} \beta)}-e^{-\tau_{c}(\mathbf{H} \alpha)}\right],
$$

where $\tau_{c}(\lambda)$ is given by equation (9) and we assume that the optical depth $\tau_{m}(\lambda)$ of the $\mathscr{N}$ clumps follows the MW or LMC extinction law.

3. The uniform scattering slab differs from model 1 in having a positive contribution to the emerging radiation from scattering, i.e., the integral in equation (6) is non zero. Therefore, equation (6) becomes an integrodifferential equation. To look for a general solution is not handy in our case, since we have to derive the effective extinction in the UV for continuous wavelength values. An approximate, but still interesting, approach considers the solution for two extreme cases: the isotropic scattering and the forward-only scattering. In the first case, $\Phi(\cos \Theta)=1$, and an approximate solution in this case is (Sobolev 1983; Natta \& Panagia 1984)

$$
I=I^{0} e^{-\tau_{\mathrm{sc}}(\lambda)} \text { with } \tau_{\mathrm{sc}}(\lambda) \simeq \sqrt{1-\omega_{\lambda}} \tau(\lambda),
$$

where $\tau(\lambda)$ is the same as model 1 . In the second case $\Phi(\cos \Theta)=2 \delta(\cos \Theta-1)$, where $\delta$ is the Dirac delta function, and yields an exact solution for equation (6) of the type

$$
\tau_{\mathrm{sc}}(\lambda)=\left(1-\omega_{\lambda}\right) \tau(\lambda)
$$

meaning that only the absorption removes photons from the line of sight, while scattering is ineffective. Intermediate cases can be approximated by a phase function expressed as (R. White 1993, private communication)

$$
\Phi(\cos \Theta)=h_{\lambda}+\left(1-h_{\lambda}\right) 2 \delta(1-\cos \Theta) ;
$$

thus,

where

$$
\tau_{\text {sc }}(\lambda)=a_{\text {eff }}(\lambda) \tau(\lambda)
$$

$$
a_{\mathrm{eff}}(\lambda)=h_{\lambda} \sqrt{1-\omega_{\lambda}}+\left(1-h_{\lambda}\right)\left(1-\omega_{\lambda}\right)
$$

and $h_{\lambda}$ is a weight parameter describing the anisotropy of the scattering at each wavelength.

If model 3 is the one which describes the dust distribution in a galaxy, the Balmer optical depth $\tau_{B}^{l}$ is given by

$$
\tau_{B}^{l}=a_{\mathrm{eff}}(\mathrm{H} \alpha) \tau(\mathrm{H} \alpha)-a_{\mathrm{eff}}(\mathrm{H} \beta) \tau(\mathrm{H} \beta) .
$$

4. The clumpy scattering slab includes the properties of both models 2 and 3 . The radiation along the line of sight receives a positive contribution from scattered light, but there are no sources of radiation inside the slab, so $\epsilon_{\lambda}=0$. The dust is organized in clumps, so $\tau_{\text {eff }}$ is given by equation (10), with

$$
\tau_{c}(\lambda)=\frac{\tau_{m}(\lambda)}{\mathscr{N}}=\frac{\tau_{\mathrm{sc}}(\lambda)}{\mathscr{N}},
$$

since now $\tau_{m}(\lambda)=\tau_{\mathrm{sc}}(\lambda)$, and $\tau_{B}^{l}$ is still given by equation (11).

5. The internal dust model has different properties from the previous four models. In this case, the sources of radiation are uniformly mixed with the dust $[\epsilon(\lambda)=$ const]. Scattering is effective in conveying photons along the line of sight, and we will use equations (15)-(16) to approximate the effect. A solution of equation (6) is

$$
I_{\lambda}=I_{\lambda}^{0} \frac{1-e^{-\tau_{\mathrm{sc}}(\lambda)}}{\tau_{\mathrm{sc}}(\lambda)}=I_{\lambda}^{0} \gamma(\lambda),
$$

and the measured Balmer optical depth is

$$
\tau_{B}^{l}=\ln \left[\frac{\gamma(\mathbf{H} \alpha)}{\gamma(\mathbf{H} \beta)}\right] .
$$

\subsection{Albedo and Phase Function}

This section aims at obtaining an analytical expression for equation (16).

For the albedo $\omega_{\lambda}$ we have adopted the list of values given in Natta \& Panagia (1984) and fitted it to a continuous function:

$$
\begin{aligned}
& \omega_{\lambda}=0.43+0.366\left\{1-e^{-\left[(y-3)^{2} / 0.2\right]}\right\} \\
& \quad \text { for } 1000 \leq \lambda \leq 3460 \AA \\
& \omega_{\lambda}=-0.48 y+2.41 \text { for } 3460<\lambda \leq 7000 \AA,
\end{aligned}
$$

with $y=\log \lambda$ (see Fig. 9). The cusp at $\lambda=3460 \AA$ will not

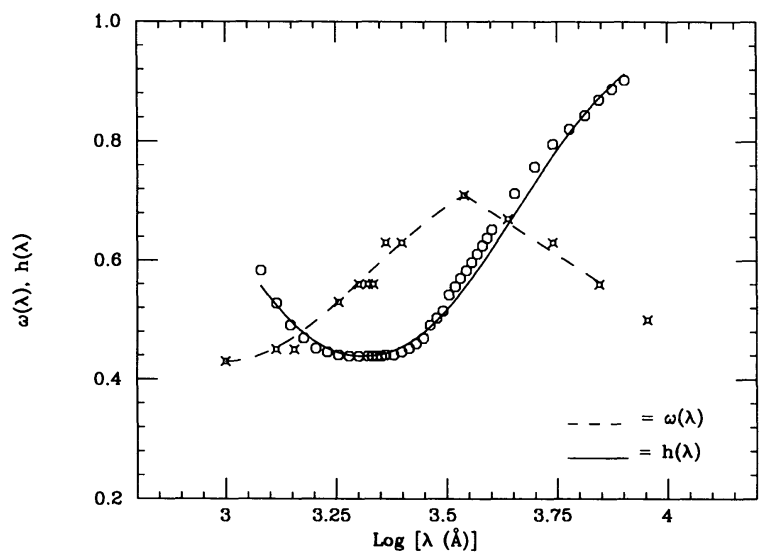

Fig. 9.-The data on the albedo $\omega_{\lambda}$ (asterisks), taken from Natta \& Panagia (1984), are reported together with the fitting function given in eq. (21) (dashed line). The values of $h_{\lambda}$ (circles), obtained from the data on the phase function from Bruzual et al. (1988) through the fitting procedure described in $\S 4.3$, are shown together with the fitting function given by eq. (22) (continuous line). 
affect our results, since we fit the UV data in the range 1250$2600 \AA$ and the optical data longward of $4500 \AA$. The difference between the fit in equations (21a)- $(21 \mathrm{~b})$ and the values given in Natta \& Panagia (1984) is 5\% at most.

The derivation of $h_{\lambda}$ has followed a somewhat more complicated procedure. Using the list of values of $g(\lambda)$ given by Bruzual et al. (1988), we have obtained the corresponding $\Phi(\lambda)$ as a Henyey-Greenstein function. Then, the best values of $h_{\lambda}$ approximating $\Phi(\lambda)$ have been derived with a least-squares fit routine. Subsequently, the set of $h_{\lambda}$ has been fitted to a continuous function in the range $1200-7000 \AA$ :

$$
h_{\lambda}=1-0.561 e^{-\left(|y-3.3112|^{2.2 / 0.17)}\right.},
$$

where $y=\log \lambda$ (see Fig. 9) .

Figure 10 shows the MW extinction law in the three cases of a uniform dust screen (eq. [8]), uniform scattering slab with isotropic scattering (eq. [12]), and with anisotropic scattering (eqs. [15]-[16]). The presence of scattering reduces not only the effective optical depth of the slab but also the strength of the $2175 \AA$ dust feature (cf. Natta \& Panagia 1984).

The major source of uncertainty in the procedure described in this section is in the derivation of the weigh function $h_{\lambda}$ from $\Phi(\lambda)$. To understand the order of magnitude of the difference between $\Phi(\lambda)$ and its approximation, equation (14), we have plotted and compared the values of $\tau_{B}^{l}$ as a function of the effective optical depth at $\mathrm{H} \beta, \tau_{\beta}=-\ln [\gamma(\mathrm{H} \beta)]$ (see eq. [19]), in an internal dust model as given by the exact solution of Mathis (1983) and by our approximation equation (14). The two solutions are reported in Figure 11, together with the case of isotropic scattering. The difference between the two curves for the anisotropic scattering (the continuous and the dot-dashed lines) is $\sim 15 \%$. The comparison involves only the optical depths of $\mathrm{H} \alpha$ and $\mathrm{H} \beta$, and therefore, it is not complete; however, on the basis of the result, we can conclude that the discrepancy between the exact solution and the approximation given in equation (15) of the optical depth $\tau_{\mathrm{sc}}(\lambda)$ is well within the measurement uncertainties of the albedo and of the phase function.

\section{COMPARISON BETWEEN MODELS AND OBSERVATIONS}

The purpose of this section is to understand which of the five models described in the previous section is more suited to

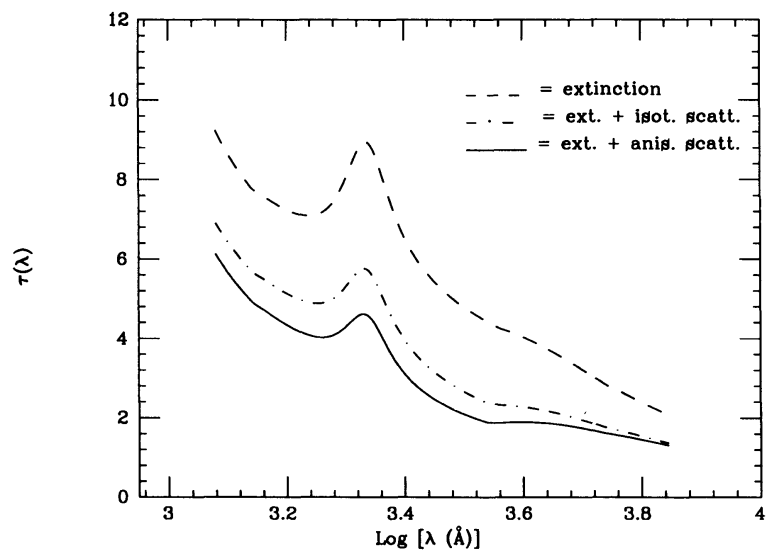

Fig. 10.-The optical depth of a uniform slab for $\tau_{B}^{l}=1$ is shown in three cases: pure extinction (eq. [8]), presence of isotropic scattering (eq. [12]), and presence of anisotropic scattering (eqs. [15]-[16]). In all the three cases a MW extinction law is assumed. The presence of scattering reduces the optical depth of the slab and also the strength of the $2175 \AA$ dust feature.

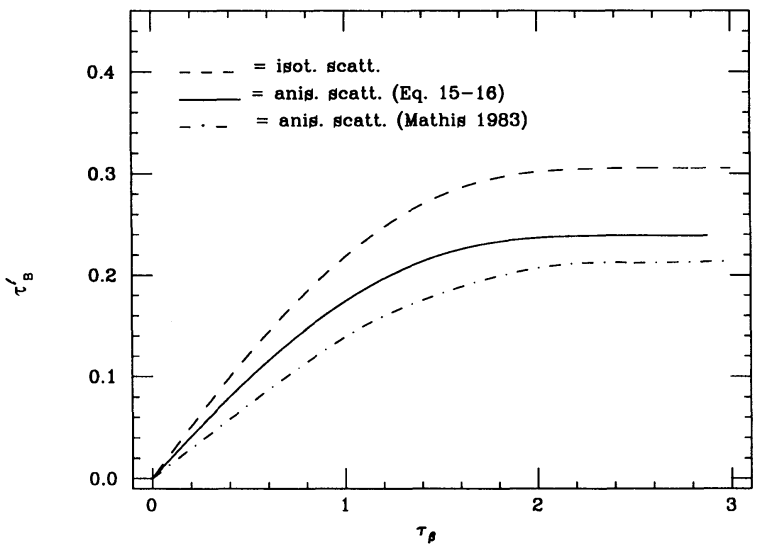

Fig. 11.-The values of the Balmer optical depth $\tau_{B}^{l}$ are shown as a function of the effective optical depth at the level of $\mathrm{H} \beta, \tau_{\beta}$, for the internal dust model (model 5) in three cases: (1) isotropic scattering (dashed line); (2) anisotropic scattering as given by the approximation in eqs. (15)-(16) (continuous line); (3) exact solution for the model (Mathis 1983; dot-dashed line). The conversion between $\tau_{B}^{l}$ and Mathis's $\tau_{\alpha \beta}$ is $\tau_{B}^{l}=0.31 \tau_{\alpha \beta}$.

explain the data, when using both the MW extinction law and the LMC extinction law.

The procedure we have chosen to compare the data in Figure $5 a$ with the models is straightforward: for different values of the Balmer optical depth $\tau_{B}^{l}$, we multiply the intrinsic (unextincted) spectrum $F(\lambda) \propto \lambda^{\beta 0}$ with the extinction curves appropriate to each of the five models and, then, fit the extincted spectrum to a power law $F(\lambda) \propto \lambda^{\beta}$ in the range 1250-2600 $\AA$, after removing the $2175 \AA$ dust feature. Due to the freedom in choosing the value of $\beta_{0}$ for the intrinsic spectrum, we adopt the best fit value from equation (4), namely $\beta_{0}=-1.71$.

In what follows is the detailed discussion of the results for each model.

\subsection{Models 1 and 3: The Uniform Dust Distribution}

In both models the dust is uniformly distributed in front of the source of ionized gas, the only difference being the positive contribution to the emerging flux due to scattering in model 3. Figure 12 reports the data and the model predictions for both the MW and the LMC extinction laws. There is only one curve which appears in agreement with the data: the case of a

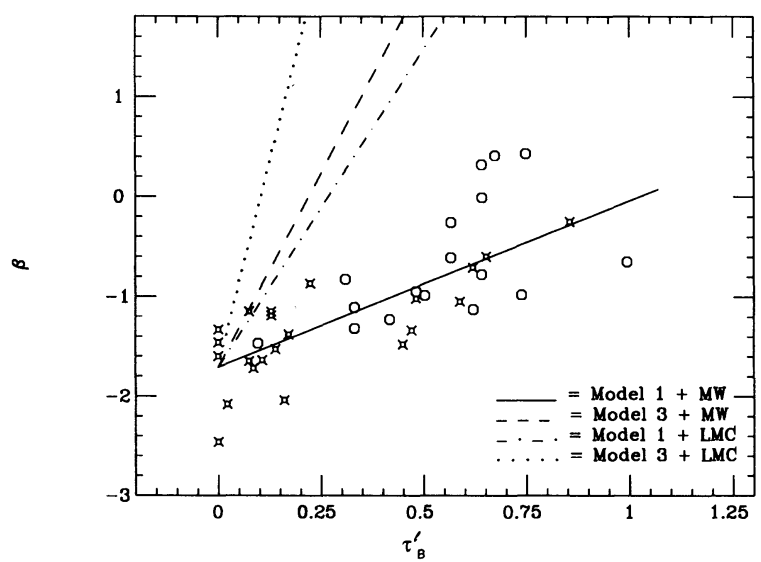

Fig. 12.-The data of $\beta$ vs. $\tau_{B}^{l}$ are compared with the curves obtained for the uniform distribution of dust in the absence (model 1) and in the presence (model 3) of scattering of photons into the line of sight. Both the cases of a MW extinction law and an LMC extinction law are considered. 
uniform dust screen (model 1) with an MW extinction law. The other three curves, the uniform screen and the scattering slap with an LMC extinction law and the scattering slab with a MW extinction law, are in clear disagreement with the data for values of $\tau_{B}^{l}>0.27$

The values for the albedo and the phase function we have fitted in $\S 4.3$ are from measurements inside the Milky Way. No multiwavelength information is available for these quantities in the case of the LMC dust type. The use of MW albedo and phase function in the case of the scattering slab plus LMC extinction law (the dotted line in Fig. 12) surely induces variations relative to the "correct" values. However, we do not expect these changes to be large enough to be the major cause of the disagreement between the theoretical line and the data.

Although the uniform dust screen plus MW extinction law appears to be in agreement with the data, we have to remember that, in the fitting procedure to obtain $\beta$, we remove the $2175 \AA$ dust feature. This feature is indeed the distinguishing mark of the MW extinction law. However, if we deredden the spectra of our galaxies according to a MW extinction law, the region around $2175 \AA$ is heavily overcorrected, especially in those cases where the values of the Balmer optical depth is large. To quantify this statement, a detailed analysis of the $2175 \AA$ dust feature has been performed. We have subtracted the appropriate linear fit $\log F(\lambda)=\beta \log (\lambda)+$ const from the 23 galaxies of our sample for which both short and long-wavelength $I U E$ spectra are available (NGC 6221, the 24th galaxy with S + L $I U E$ spectrum, has not been included in the analysis, because its spectrum is too noisy to provide reliable results). The region around $2175 \AA$ of each residual spectrum has been fitted with a Drude profile given by (Fitzpatrick \& Massa 1988)

$$
\Delta \log F(\lambda)=\eta \frac{x^{2}}{\left(x^{2}-x_{0}^{2}\right)^{2}+\gamma^{2} x^{2}},
$$

where $x=\lambda^{-1}\left(\mu \mathrm{m}^{-1}\right), x_{0}=\lambda_{0}^{-1}=4.6 \mu \mathrm{m}^{-1}$ is the central wavelength of the dust bump, and $\gamma=0.95 \mu \mathrm{m}^{-1}$ is its FWHM. The quantity $\eta$ is a function of both the "intrinsic depth" of the bump and the Balmer optical depth $\tau_{B}^{l}$ (i.e., the amount of reddening in the spectrum). $\eta$ is expected to be zero for $\tau_{B}^{l}=0$ and to become more negative as $\tau_{B}^{l}$ increases. In addition, the larger the bump in the extinction curve, the more negative the value of $\eta$ for constant values of $\tau_{B}^{l}$.

Figure 13 shows the measured values of $\eta$ for our galaxies as a function of $\tau_{B}^{l}$. The typical $1 \sigma$ internal error bar for $\eta$ is also shown, although the external errors probably give a much larger uncertainty. A linear fit to the data yields

$$
\eta=(-0.07 \pm 0.05) \tau_{B}^{l}+(0.01 \pm 0.02) \text {. }
$$

For comparison, the curves expected for a uniform dust screen (model 1) with a MW and an LMC extinction law are plotted. It is clear from this figure that model 1 plus a MW extinction curve does not fit the (un)observed dust bump at $2175 \AA$.

The conclusion from the comparison between the data and models 1 and 3 is that the two models are not able to explain the data, when MW or LMC extinction laws are adopted.

\subsection{Models 2 and 4: The Clumpy Dust Distribution}

Model 2 differs from model 4, as in the previous case of models 1 and 3 , by the presence in model 4 of scattering of photons into the line of sight.

These two models of clumpy dust are probably closer to a realistic description of the dust distribution inside a galaxy than the previous two models of uniform dust distribution,

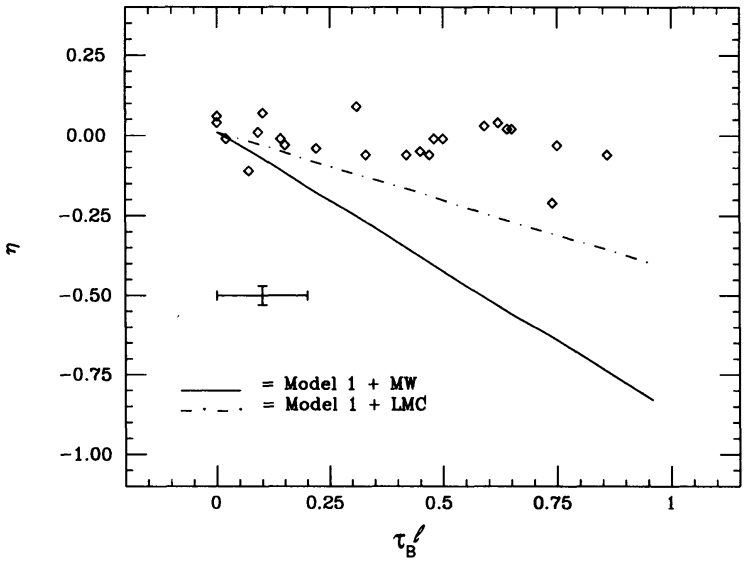

FIG. 13.-The "depth" of the $2175 \AA$ dust bump, $\eta$ (see eq. [23]), is shown as a function of the Balmer optical depth $\tau_{B}^{l}$ for our data (diamonds), and for the case of a uniform dust screen with MW (solid line) and LMC (dot-dashed line) extinction law. The formal $1 \sigma$ error bar on the data is also shown. Our sample contains 24 galaxies for which short and long wavelength IUE spectra are available, but NGC 6221 has been excluded from the analysis because of its noisy spectrum.

since dust appears inhomogeneously distributed in $\mathrm{H}$ II regions and in galaxies in general (e.g., Waller, Gurwell, \& Tamura 1992). Therefore, the models deserve careful discussion.

With respect to the previous case of uniform distribution, models 2 and 4 have an additional free parameter: the average number $\mathscr{N}$ of clumps along the line of sight. A large value for $\mathscr{N}$ gives $\tau_{m} \simeq \tau_{\text {eff }}$, and models 2 and 4 converge to the uniform cases 1 and 3, respectively. So, we are interested in cases where $\mathscr{N} \lesssim 20$ (see Fig. $15 a$ ).

The effective optical depth $\tau_{\text {eff }}$ in the clumpy models (see eq. [10]) has the characteristic of reaching the asymptotic value $\mathscr{N}$ for large values of the optical depth $\tau_{c}$ of the clumps. Obviously, this effect becomes more evident as $\mathscr{N}$ decreases (eqs. [9] and [10]), since a lower value of the average optical depth $\tau_{m}$ is necessary to reach the asymptote. The implication is that the extinction becomes a "gray extinction" for large optical depths: therefore $\tau_{B}^{l} \rightarrow 0$ and $\beta \rightarrow \beta_{0}$. This explains both the behavior of $\tau_{B}^{l}$ as a function of $\tau_{m}$ for small values of $\mathcal{N}$ in Figure 14 and the increasingly nonlinear behavior of the curve

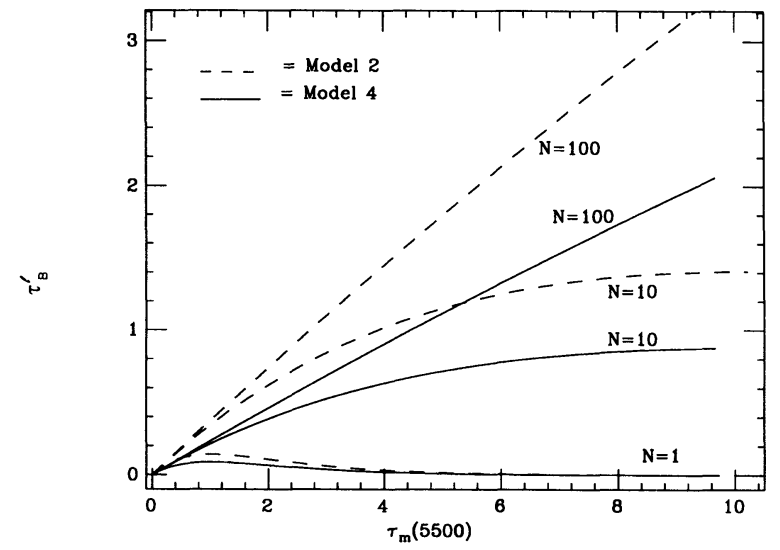

Fig. 14.-The values of the Balmer optical depth $\tau_{B}^{l}$ are shown as a function of the average optical depth $\tau_{m}$ at $5500 \AA$ for clumpy dust distribution with $\mathscr{N}=100,10,1$, both the absence (model 2) and in the presence (model 4) of scattering of photons into the line of sight. The MW and LMC extinction laws coincide in the optical wavelength range, therefore, the derived $\tau_{B}^{l}$ are the same for both curves. 

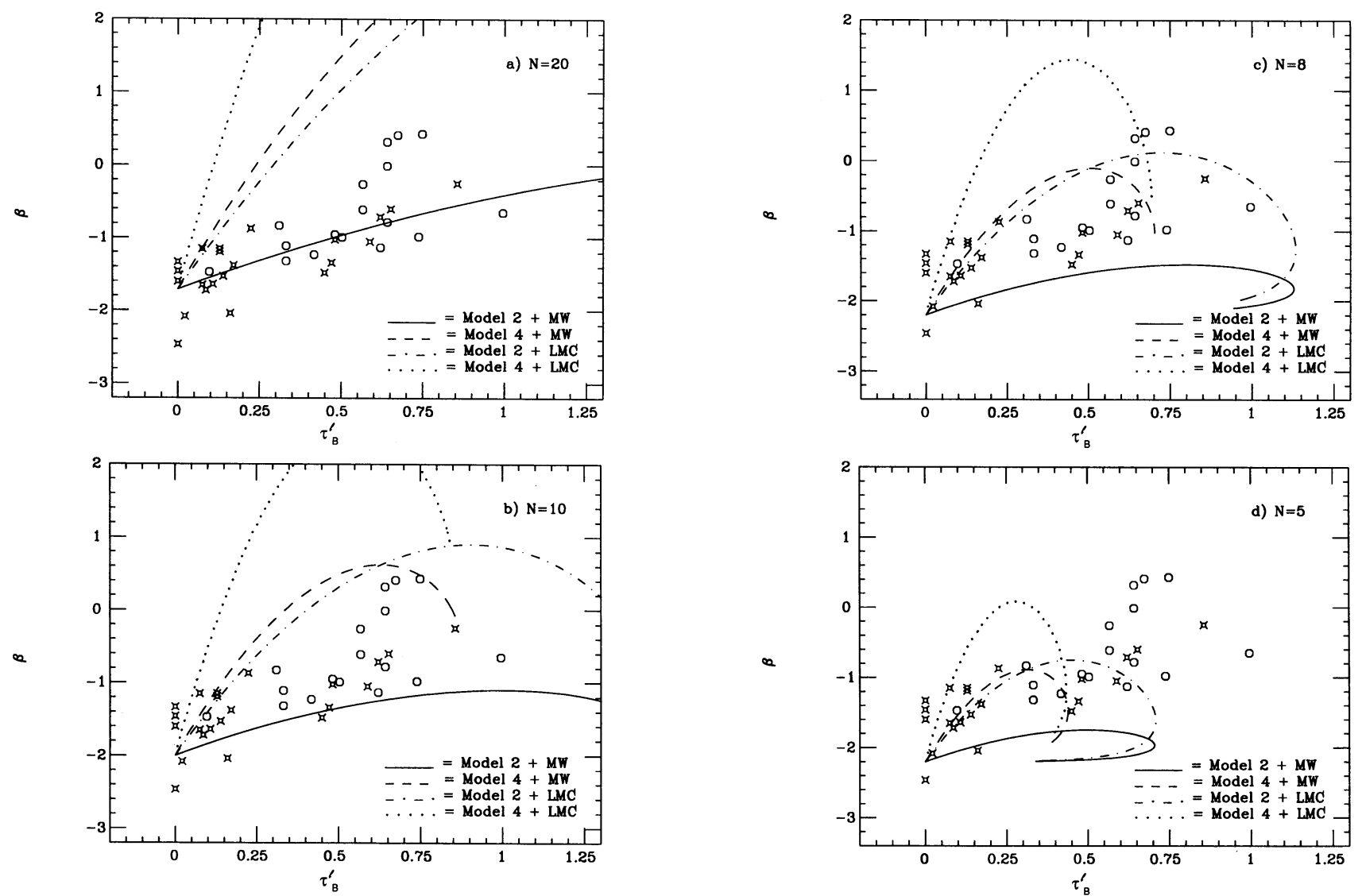

Fig. 15.-The data on $\beta$ as a function of $\tau_{B}^{l}$ are compared with the expected trend for clumpy dust distributions with different average number of clumps along the line of sight: $(a) \mathcal{N}=20,(b) \mathcal{N}=10,(c) \mathscr{N}=8,(d) \mathscr{N}=5$. Both the presence (model 4) and the absence (model 2) of scattering of photons into the line of sight are considered. The MW and LMC extinction curves are used.

$\beta$ versus $\tau_{B}^{l}$ in Figure 15 as $\mathscr{N}$ decreases in value (in Fig. 15a, $\mathscr{N}=20 ;$ Fig. $15 b, \mathscr{N}=10 ;$ Fig. $15 c, \mathscr{N}=8 ;$ Fig. $15 d$, $\mathscr{N}=5)$.

We have attempted to fit models 2 and 4 to the data of Figure $5 a$ by leaving both the values of $\beta_{0}$ and of $\mathscr{N}$ as free parameters and including both the MW and LMC extinction laws, but no fit gives satisfactory results (see Fig. 15). Although model 2 plus the Milky Way extinction law and $\mathscr{N}=20$ appears to be in agreement with the data, the $2175 \AA$ dust feature is stronger than allowed by the observed spectra (see $\S 5.1$ and Fig. 13).

The conclusion is that not even the models of clumpy dust appear to explain the correlation between $\beta$ and $\tau_{B}^{l}$.

\subsection{Model 5: The Internal Dust}

The model of dust/gas homogeneously mixed is an approximation for the presence of dust between the ionizing stars (internal dust). It is important to verify if the correlation between $\beta$ and $\tau_{B}^{l}$ can be explained by a model of internal dust, since the presence of internal dust in a cluster of stars is highly reasonable.

The first difficulty arises when analyzing the behavior of $\tau_{B}^{l}$ as a function of the optical depth at $\mathrm{H} \beta, \tau_{\beta}$ (see Fig. 11): $\tau_{B}^{l}$ reaches an asymptote with value $\simeq 0.24$. Therefore, it is difficult to explain why there are values $\tau_{B}^{l}>0.24$ in Figure $5 a$.

Analogously, $\beta$ reaches an asymptote (see Fig. 16), and most of the points in the $\beta$ versus $\tau_{B}^{l}$ plane are unexplained. The overall disagreement between the model and the data is in line with the idea that internal dust does not determine the shape of the extincted SED of a galaxy, but mainly removes the contribution of the most internal (and most extincted) stars from the emerging flux (see Witt et al. 1992).

To conclude, although there is still the possibility that at least some fraction of the total dust in our galaxies is internal dust, internal dust by itself does not account for the correlation between the power-law index of the UV spectra and the Balmer line ratio in our sample galaxies.

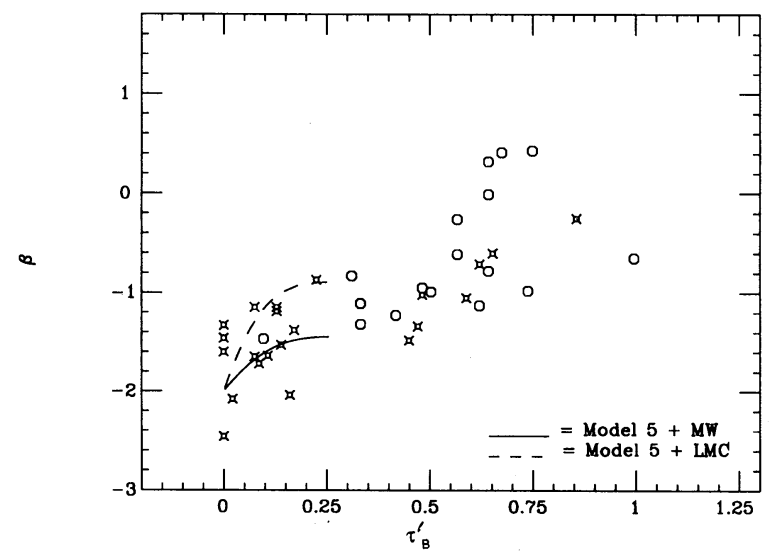

FIG. 16.-The data on $\beta$ vs. $\tau_{B}^{l}$ are compared with the curves for the internal dust model (model 5), in both cases of MW and LMC extinction laws. 


\section{THE EXTINCTION LAW}

The inability of the five models for the dust configuration to account for the correlation between $\beta$ and $\tau_{B}^{l}$ has several possible explanations: (1) The dust configuration is more complex than we can describe with the models discussed in the previous section; (2) the MW and LMC extinction laws do not apply to our sample; (3) although Figure $5 a$ tells us that the UV powerlaw index is proportional to the Balmer optical depth $\tau_{B}^{l}$, it does not give the constant of proportionality: if it is different from unity, the disagreement with the models can be partially explained (see $\S 6.4$ ).

In any case, the existence of a correlation between $\beta$ and $\tau_{B}^{l}$ is evidence that the dust extinction in the UV is linked to the dust extinction which affects the ionized gas (although it has been pointed out by other authors that the Balmer line ratio gives an overestimation of the extinction in the UV, e.g., Fanelli et al. 1988). We will use this property to derive an extinction law suitable for the galaxies in our sample and compare it with the ones we can obtain from the five models of $\S 4$. Section 6 is devoted to the derivation of the extinction law from our sample galaxies and its comparison with theoretical models for the dust distribution.

The procedure described in the following subsections is a statistical generalization of the method presented in Kinney et al. (1994b) for the case of two starburst galaxies, NGC 7552 and NGC 5236.

\subsection{The Template Spectra and the $2175 \AA$ Dust Feature}

The problem in using galaxies instead of stars for deriving extinction laws is that spectra of galaxies are product of contributions from stellar populations of different ages, which, for instance, determine the intensity of the jump around $3500 \AA$ and, in the case of starbursts, may affect the UV power-law index. However, the availability of a relatively large sample allows us to average out the effects of different stellar populations within similar values of the extinction.

In order to obtain "galaxy spectra with average stellar populations," that will be called from now on "templates" (see Kinney, Calzetti, \& Storchi-Bergmann, 1994a), we have divided the sample in bins of $\Delta \tau_{B}^{l}=0.11$ and averaged the spectra within each bin. The galaxies which are included within each bin of extinction are listed in Table 3. The averaging procedure goes through the following steps: (1) correction of the flux for the foreground Milky Way extinction; (2) shift of the spectrum to the rest-frame wavelengths; (3) average with the other spectra within the same bin of extinction after weighting each spectrum by its signal-to-noise ratio. The procedure is explained in Kinney et al. (1994a). Some of the galaxies in the original sample have not been included in the elaboration of the templates; they have been excluded for various reasons: NGC 1705, because it is classified as a poststarburst and the properties of its UV stellar population differ from the ones of the other galaxies; NGC 1569, because its spectrum is strongly affected by the foreground Milky Way extinction; NGC 6221, because the signal-to-noise ratio of its UV spectrum is among the lowest in our sample. In addition, no bins of extinction beyond $\tau_{B}^{l}=0.75$ have been considered because they are not populated enough to give reliable average spectra.

Figure 17 shows the spectra of the templates and Figure 18, the position of the templates in a $\beta$ versus $\tau_{B}^{l}$ plot. In Table 3 and in Figure 17, the different templates have been labeled with increasing numbers for increasing values of the average extinc-
TABLE 3

TEMPlate SPECTRA

\begin{tabular}{|c|c|}
\hline Range in $E(B-V)_{i}$ & Galaxy Name \\
\hline \multicolumn{2}{|l|}{ Template 1} \\
\hline$\tau_{B}^{l} \leq 0.11 \ldots \ldots \ldots \ldots \ldots \ldots$ & $\begin{array}{l}\text { Tol 1924-416 } \\
\text { NGC } 1510 \\
\text { NGC } 1800 \\
\text { NGC } 1140 \\
\text { Mrk } 66 \\
\text { NGC } 7250 \\
\text { NGC } 5253 \\
\text { Haro } 15\end{array}$ \\
\hline \multicolumn{2}{|l|}{ Template 2} \\
\hline $0.12 \leq \tau_{B}^{l} \leq 0.23 \ldots$ & $\begin{array}{l}\text { UGC } 9560 \\
\text { NGC } 3125 \\
1941-543 \\
\text { Mrk } 357 \\
\text { UGCA } 410 \\
\text { NGC } 6052\end{array}$ \\
\hline \multicolumn{2}{|l|}{ Template 3} \\
\hline $0.27 \leq \tau_{B}^{l} \leq 0.38 \ldots$ & $\begin{array}{l}\text { Mrk } 542 \\
\text { NGC } 3049 \\
\text { NGC } 5236\end{array}$ \\
\hline \multicolumn{2}{|l|}{ Template 4} \\
\hline $0.42 \leq \tau_{B}^{l} \leq 0.54 \ldots \ldots \ldots \ldots \ldots$ & $\begin{array}{l}\text { NGC } 7673 \\
\text { NGC } 7793 \\
\text { NGC } 7714 \\
\text { Mrk } 499 \\
\text { NGC } 5996\end{array}$ \\
\hline \multicolumn{2}{|l|}{ Template 5} \\
\hline $0.55 \leq \tau_{B}^{l} \leq 0.64 \ldots \ldots \ldots \ldots \ldots$ & $\begin{array}{l}\text { NGC } 4385 \\
1050+04 \\
\text { NGC } 6090 \\
\text { ICI } 1586 \\
\text { NGC } 6217 \\
\text { IC } 214\end{array}$ \\
\hline \multicolumn{2}{|l|}{ Template 6} \\
\hline $0.65 \leq \tau_{B}^{l} \leq 0.75 \ldots \ldots \ldots \ldots \ldots$ & $\begin{array}{l}\text { NGC } 5860 \\
\text { NGC } 1313 \\
\text { NGC } 1672 \\
\text { NGC } 3256 \\
\text { NGC } 7552\end{array}$ \\
\hline
\end{tabular}

tion. The spectra in Figure 17 show that increasing values of the Balmer optical depth correspond to increasing values of $\beta$. However, there is little evidence for the presence of the $2175 \AA$ dust feature in any of the templates (cf. Fig. 13).

\subsection{The Derivation of the Extinction Law}

The dependence of $\beta$ on $\tau_{B}^{l}$ is well described by a linear correlation (see Fig. 5): in fact, the Pearson linear correlation test gives a coefficient $r_{p}=0.75$. Therefore, we will use the quantity $\tau_{B}^{l}$ as the independent parameter in the derivation of the extinction law. This assumption implies that the dust behaves as a uniform screen (models 1 or 3 ) in front of the emitting source. A clumpy dust model with a low average number of clumps along the line of sight, $\mathscr{N} \lesssim 10$, gives a nonlinear dependence between $\beta$ and $\tau_{B}^{l}$ (see $\S 5$ ). A strongly nonlinear dependence is excluded by the data and a mild nonlinearity would be hidden by the intrinsic spread of the data; in fact, the clumpy dust model can still be in agreement with the 


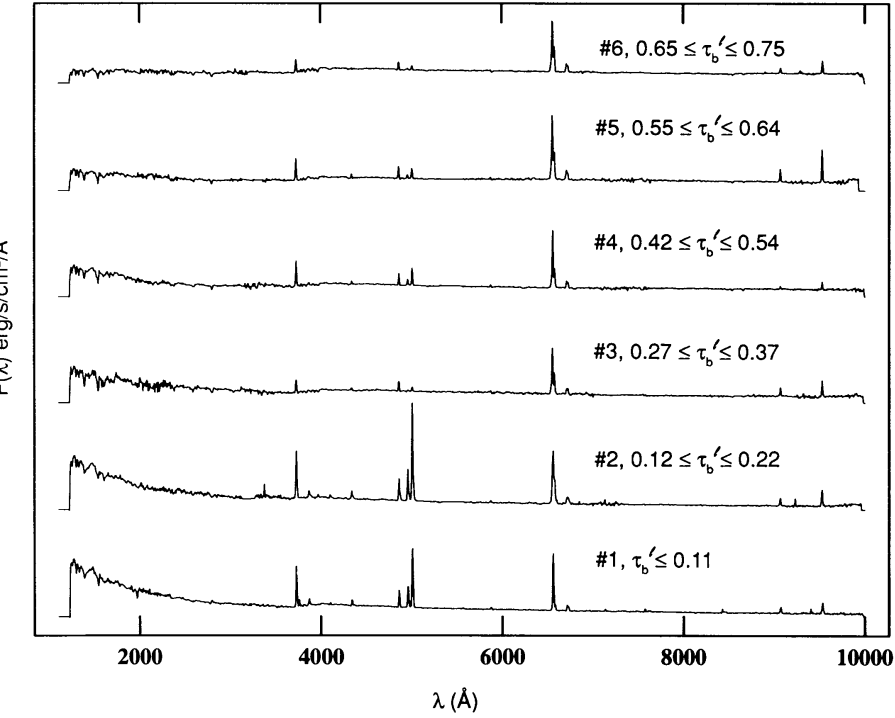

FIG. 17.-The spectra of the six templates are shown for increasing values of the extinction parameter $\tau_{B}^{l}$, from the bottom to the top of the figure.

data if $\mathscr{N}>10$ (see Figs. $15 a-15 b$ ). The assumption of linear dependence between $\beta$ and $\tau_{B}^{l}$ allows the minimum number of hypotheses to be introduced.

For the purpose of deriving the extragalactic extinction law, the six templates have been smoothed in bins of $50 \AA$ and have been associated with the average values $\tau_{B}^{l}=0.05,0.16,0.32$, $0.48,0.59,0.70$, respectively.

Template $1\left(\tau_{B}^{l}=0.05\right)$ is used as reference spectrum, against which the other five will be compared. Therefore, from equation (1), we can define

$$
\tau_{n}(\lambda)=-\ln \frac{F_{n}(\lambda)}{F_{1}(\lambda)}
$$

where $\tau_{n}(\lambda)$ is the dust optical depth corresponding to the template $n$ with flux $F_{n}(\lambda)$. The Balmer optical depth $\tau_{B}^{l}$ parameterizes the templates, and we can define a rescaled optical depth

$$
Q_{n}(\lambda)=\frac{\tau_{n}(\lambda)}{\delta \tau_{B n}^{l}},
$$

where $\delta \tau_{B n}^{l}=\tau_{B n}^{l}-\tau_{B 1}^{l}$ is the difference between the Balmer

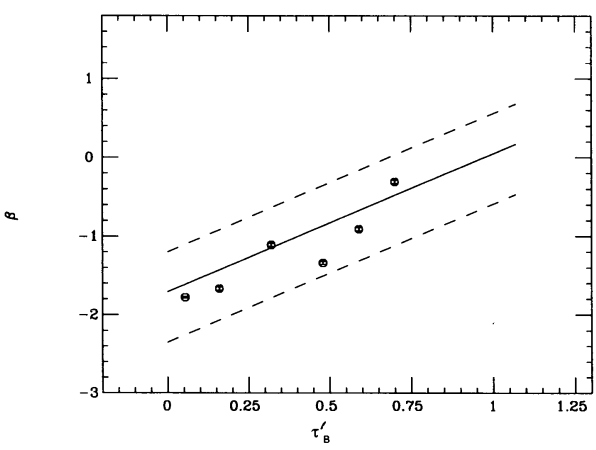

Fig. 18. - The UV power-law index $\beta$ is shown as a function of the Balmer optical depth $\tau_{B}^{l}$ for the six templates discussed in the text. The $1 \sigma$ error bar for each power-law index is the size of the symbols. The best-fit line (continuous line) and the envelopes (dashed lines) from Fig. $5 a$ are also reported for comparison. optical depths of the templates $n$ and 1. The differences between the Balmer optical depths are $\delta \tau_{B n}^{l}=0.11,0.27,0.43$, $0.54,0.65$ for the templates $n=2,3,4,5,6$, respectively. In order to minimize the contribution from spectral fluctuations, we will consider only $Q_{n}(\lambda)$ with $n=3,4,5,6$. In the case of the template 2 , the difference $\delta \tau_{B 2}^{l}=0.11$, is quite small and fluctuations dominate the trend.

Since the templates are spectral averages of galaxies at different distances and with different luminosities, equation (24) will give us information on the selective extinction, but not on the total extinction, implying that the zero point of $Q_{n}(\lambda)$ is arbitrary. We have chosen as zero point $Q_{n}(5500)=0.0$ and, after weighting each function by its associated $\delta \tau_{B n}^{l}$, we have averaged the $Q_{n}(\lambda)$ to produce $Q_{e}(\lambda)$, that we will call the effective extinction law. Figure 19 reports the four $Q_{n}(\lambda)$ with $n=3,4,5$, 6 plotted together with $Q_{e}(\lambda)$. The fact that the four $Q_{n}(\lambda)$ show a remarkable agreement in their behavior not only in the UV but also in the optical wavelength range confirms that (1) $\tau_{B}^{l}$ is an appropriate parameter to characterize the effective extinction law; (2) the sample of galaxies we have considered is large enough to yield templates, namely, galaxies with "average stellar populations."

The effective extinction law $Q_{e}(\lambda)$ has been fitted in the range $1250-8000 \AA$ to a third-degree polynomial as a function of $x=1 / \lambda\left(\mu \mathrm{m}^{-1}\right)$ :

$$
Q_{e}(x)=-2.156+1.509 x-0.198 x^{2}+0.011 x^{3} .
$$

The formal $1 \sigma$ uncertainty on the parameters is about $2 \%$. Figure 20 shows the fit overplotted to $Q_{e}(\lambda)$. It is evident that the $2175 \AA$ dust feature is absent.

Figure 21 shows the extinction curve we have just derived in comparison with the MW and LMC extinction laws, after imposing as zero point for the latter two the value $k(5500)=0.0$ and transforming $k(\lambda)$ to $Q(\lambda)$ through the relation

$$
Q(\lambda)=\frac{k(\lambda)}{k(\mathrm{H} \beta)-k(\mathrm{H} \alpha)} .
$$

The effective extinction law given by equation (25) is clearly more gray than the stellar extinction laws (MW and LMC), and there is no evidence of a $2175 \AA$ dust feature. Figure 21 shows also, for comparison, the effective extinction law derived by Kinney et al. (1994b) from the spectral energy distributions

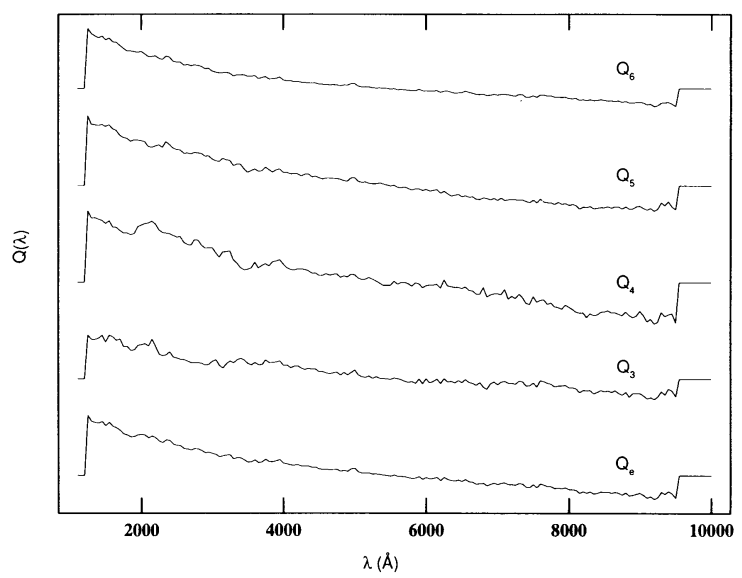

FIG. 19.- The effective extinction law $Q_{e}(\lambda)$ is compared with the extinction law derived from each single template $Q_{n}(\lambda)$, with $n=3,4,5,6$. 


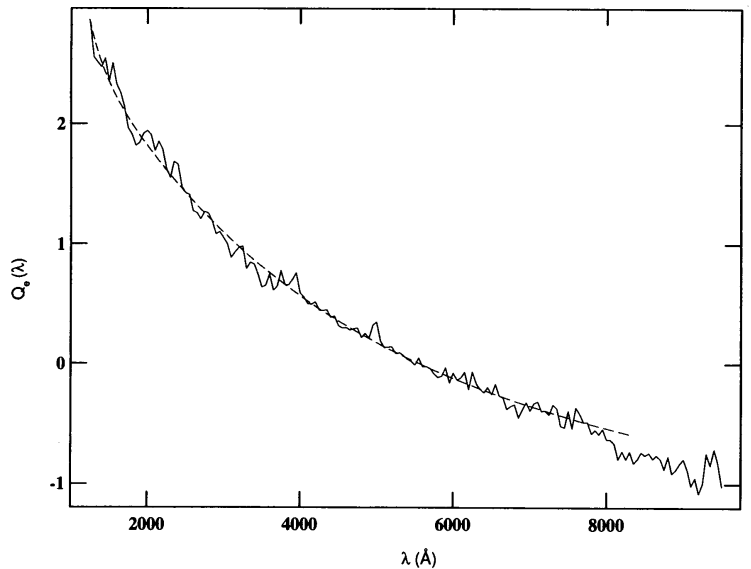

FIG. 20.-The effective extinction law $Q_{e}(\lambda)$ is shown together with its best fit overplotted in the wavelength range $1250-8000 \AA$.

of the two galaxies NGC 7552 and NGC 5236. These two galaxies have been selected because the features in their spectra indicate that the stellar populations contributing to the UV and optical emission are similar and, therefore, any difference between the two spectral energy distributions can be attributed to dust extinction. Taking into account the difference in method used to derive equation (25) and Kinney et al.'s curve, the two results can be considered consistent.

\subsection{The Extinction of the Stellar Continuum}

Equation (25) provides a way to determine the selective dust extinction of the integrated stellar continuum in external galaxies. In fact, the selective optical depth of a star-forming galaxy can be derived by measuring the difference in optical depth of the Balmer emission lines, $\tau_{B}^{l}$ and by multiplying this quantity by $Q_{e}(\lambda)$.

An interesting property of $Q_{e}(\lambda)$ is that the difference $\tau_{B}^{c}$ between the optical depths of the continuum underlying the Balmer lines $\mathrm{H} \alpha$ and $\mathrm{H} \beta$ is given by

$$
\tau_{B}^{c}=\left[Q_{e}(\mathrm{H} \beta)-Q_{e}(\mathrm{H} \alpha)\right] \tau_{B}^{l}=(0.5 \pm 0.11) \tau_{B}^{l},
$$

where the uncertainty is given by the root mean square deviation of the four $Q_{n}(\lambda)$ from the mean value (for an independent

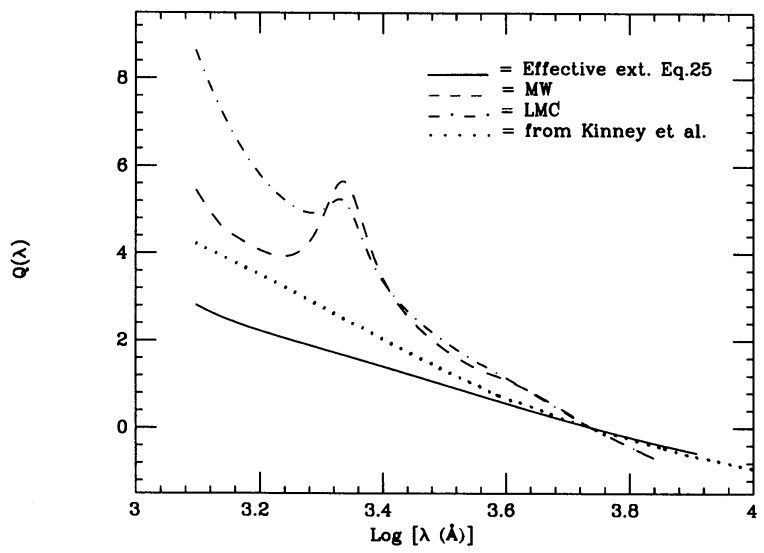

Fig. 21.-The extinction law derived in this work (eq. [25], continuous line) is compared with the Milky Way (dashed line) and the LMC (dot-dashed line) extinction laws. The extinction law derived by Kinney et al. (1994b) is also shown (dotted line). The zero point of the four curves is arbitrary and has been chosen to be the value $Q(5500)=0.0$. derivation of eq. [26], see Storchi-Bergmann et al. 1994a). Such a difference is not unexpected, since, as discussed in $\S 3.6$, the continuum and the emission lines are not necessarily due to the same stars. The drifting of stars from their parent clouds toward regions of low extinction favors long-living stars, namely stars which contribute to the optical continuum and the UV longward of $1200 \AA$ : one plausible scenario is that the hot ionizing stars sit close to or in dusty clouds, while the colder stars, which contribute most of the optical and part of the UV continuum, are far from the dusty regions. The relation given by equation (26) is entirely due to the fact that we are determining the extinction curve from extended regions of galaxies instead of from single stars.

\subsection{The Comparison with the Five Dust Models}

The fact that hot ionizing stars are, from the previous reasoning, evidently more extincted than the stars which provide the continuum, has consequences also for the five models described in $\S 4$.

We investigate here whether or not the dust models described in $\S 4$ are in agreement with the data in Figure $5 a$ when we fit $\beta$ by adopting the relation given by equation (26) between the difference in the optical depth of nebular lines and the difference in the optical depth of the stellar continuum. Model 5, the internal dust, will not be considered further because of the restricted range of values for $\tau_{B}^{l}$ resulting from that model. There are no analogous restrictions for the other four models, which will be considered for additional analysis.

Figure $22 a$ shows the models 1 and 3 in comparison with the data in the hypothesis that $\tau_{B}^{c}=0.5 \tau_{B}^{l}$. The MW extinction law is too flat, while the LMC extinction law is still too steep to explain the UV power-law indexes. Figure $22 b$ shows the comparison between the data and models 2 and 4 . In the case of clumpy dust we have assumed that the optical depth $\tau_{c}$ of the single clump is constant, so equation (26) implies that the average number of clumps in front of the generic stellar population is one-half of the average number of clumps in front of the ionized gas. Although the overlap between the models and the data has somewhat improved (especially in the case of the LMC extinction law), the fit is still unsatisfactory. As a further check, we have compared the observed "depth " $\eta$ of the 2175 $\AA$ dust bump (eq. [23]) with the expected "depth" in the case of model 2 with $\mathcal{N}=10$ plus an LMC extinction law and $\tau_{B}^{c}=0.5 \tau_{B}^{l}$. The results are shown in Figure 23; although the agreement between the data and the model has improved relative to Figure 13, the fit is still unacceptable.

The conclusion is that, within the limits of the five models considered, the MW and the LMC extinction laws are not suitable to deredden the UV spectra of extended regions of galaxies, because either the overall slope is different, or the $2175 \AA$ dust feature is too strong to agree with the data.

\subsection{Extinction Correction of Absorption and Emission Lines}

Up to this point we have dealt with the integrated stellar continuum of galaxies. The next question is how to correct for extinction the absorption and emission lines.

In the case of starbursts, we can identify three kinds of lines: stellar and interstellar absorption lines and nebular emission lines. The stellar absorption lines experience the same dust effects as the stellar continuum, so that the considerations presented above are valid also for the stellar lines. Therefore, the equivalent width of the stellar lines is independent of the extinction. The same reasoning is valid for the interstellar 

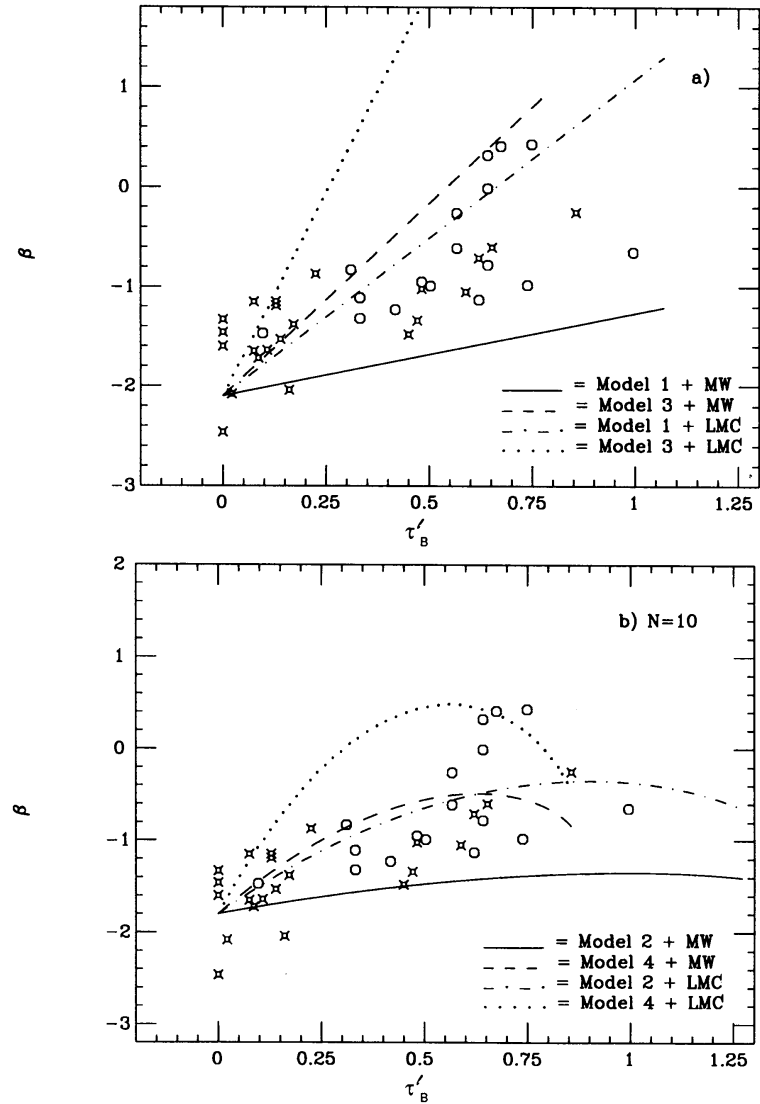

FIG. 22.-The data of $\beta$ as a function of $\tau_{B}^{l}$ are compared with models 1-4 in the hypothesis that the extinction of the stellar continuum is one-half of the extinction of the nebular lines, $\tau_{B}^{c}=0.5 \tau_{B}^{l}$. Both the MW and the LMC extinction laws are used. (a) Comparison of the data with models 1 and 3 assuming $\beta_{0}=-2$.1. (b) Comparison of the data with models 2 and 4 assuming $\mathscr{N}=10$ and $\beta_{0}=-1.8$.

absorption lines, since these lines are produced by the ISM in front of the same stellar populations which produce the stellar lines.

A different approach is necessary for the nebular emission lines. As shown above, the most obvious interpretation for the factor 2 between $\tau_{B}^{l}$ and $\tau_{B}^{c}$ is that the nebular lines come from dustier regions than the average stellar continuum. The most direct consequence is that the equivalent width of the emission line is a function of the extinction, namely,

$$
\mathrm{EW}_{\mathrm{obs}}=\mathrm{EW}_{\mathrm{int}} e^{-0.5 \tau_{B} l}
$$

where $\mathrm{EW}_{\mathrm{obs}}$ is the observed value of the equivalent width, $\mathrm{EW}_{\text {int }}$ is the intrinsic (unreddened) value of the equivalent width of the line. Testing equation (27) is not an easy task, since the strength of the emission lines depends on the physical and chemical conditions of the nebula. Hydrogen lines are not sensitive to the chemical abundances, but the level of ionization of the nebula determines the intensity of the lines. In addition, the contribution to the underlying continuum from stellar populations not directly involved in the ionization of the lines is not constant, and this introduces another variable in the value of the equivalent width.

However, our templates should approximate the condition of an "average stellar population," so that we can check the compatibility of equation (27) with observations by using the templates. We measure the equivalent width of $\mathrm{H} \beta$ and $\mathrm{H} \alpha$ in

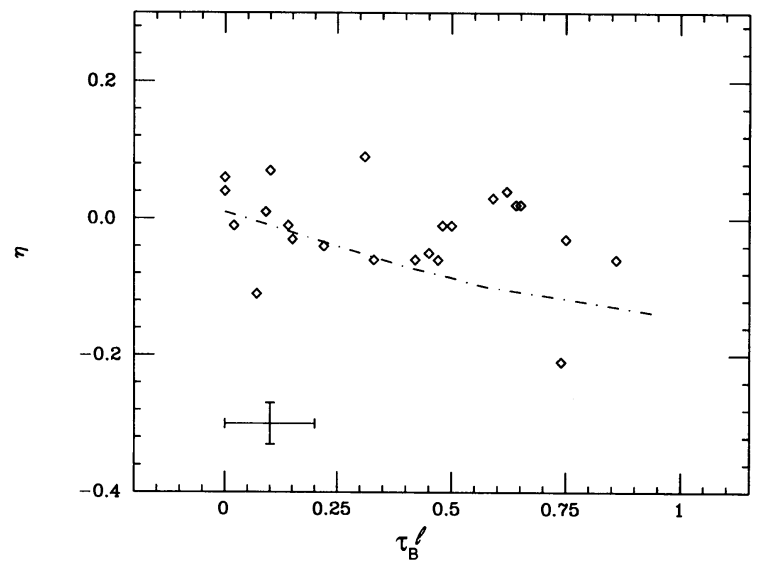

FIG. 23.-The data on the "depth" of the $2175 \AA$ dust bump, $\eta$, are compared with the expected trend in the case of a uniform clumpy dust screen (model 2) with $\mathscr{N}=10$, an LMC extinction law and $\tau_{B}^{c}=0.5 \tau_{B}^{l}$. The fit is unsatisfactory, although the agreement between data and model has improved relative to the models in Fig. (13).

the reference spectrum (template 1) and in each of the templates $n$, also accounting for an average underlying stellar absorption of $2 \AA$, and compare the ratio $\mathrm{EW}_{n} / \mathrm{EW}_{1}$ with the expectation from equation (27). The results are reported in Figure 24. The $1 \sigma$ error bars there reported are nominal, and the actual uncertainties are probably larger, since the corrections for the underlying stellar absorption are uncertain and, in the case of $\mathrm{H} \alpha$, the deblendling with the $\mathrm{N}$ II lines becomes difficult at low extinctions. Nevertheless, Figure 24 shows that there is compatibility between the predicted exponential behavior and the measured values for the ratio of the observed to the intrinsic equivalent width.

It is also interesting that, in those few cases where the Ly $\alpha$ emission has been detected, the equivalent width of the line shows a decreasing trend as a function of increasing values of the oxygen abundance of the galaxy (Charlot \& Fall 1993). The trend is well described by an exponential of the type given in

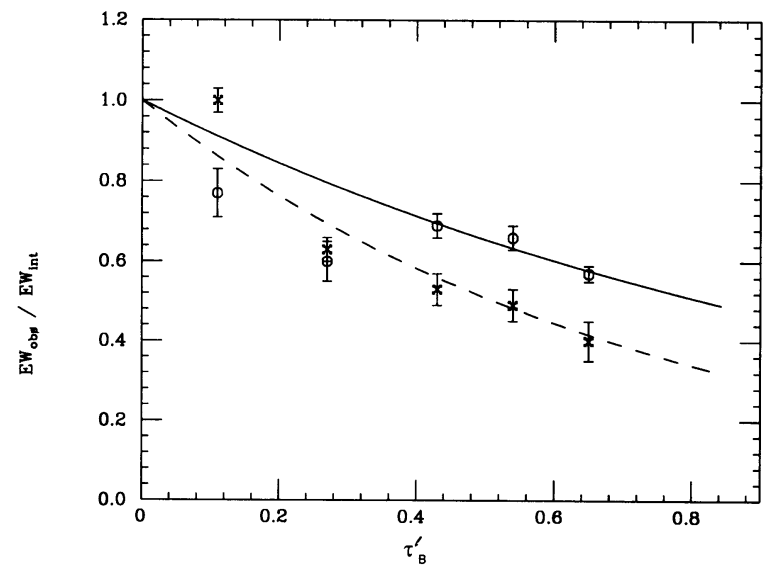

FIG. 24. - The variation of the ratio of the observed equivalent width to the intrinsic equivalent width $\mathrm{EW}_{\text {obs }} / \mathrm{EW}_{\text {int }}$ as a function of $\tau_{B}^{l}$ for $\mathrm{H} \alpha$ and $\mathrm{H} \beta$. The continuous line and the dashed line are the predictions for $\mathrm{H} \alpha$ and $\mathrm{H} \beta$, respectively, based on the extinction law eq. (25) and on the model of differential extinction between the nebular emission lines and the optical stellar continuum. The circles and the crosses are measured ratios of the attenuated to unattenuated equivalent widths $\left(\mathrm{EW}_{n} / \mathrm{EW}_{1}\right.$ of the $n$th template to the template 1) for both $\mathrm{H} \alpha$ and $\mathrm{H} \beta$, respectively. The $1 \sigma$ error bars on the measurements are also indicated. 
equation (27), if we admit a correlation between $\tau_{B}^{l}$ and $\mathrm{O} / \mathrm{H}$ (see $\S 3.3$ ). A detailed comparison is not possible, due to the necessity of assuming a relation between $\tau_{B}^{l}$ and $\mathrm{O} / \mathrm{H}$ and due to the multiple effects which can determine the equivalent width of the Ly $\alpha$ line, ranging from the nonnegligible contribution of the underlying stellar absorption (Valls-Gabaud 1993) to the possible presence of an enhanced dust absorption due to multiple scattering with H I (Neufeld 1990).

\section{SUMMARY AND CONCLUSIONS}

The present study provides an analytical formulation of the effects of dust extinction in extended regions of galaxies and an effective extinction law for correcting the observed UV and optical spectral continua, expressed in terms of easily observable quantities, such as the Balmer line ratio $\mathrm{H} \alpha / \mathrm{H} \beta$.

The large statistical sample has allowed us to average the contributions due to differences in the stellar populations of the various galaxies and, therefore, to distinguish this effect from the average effect of the dust extinction.

The comparison between our data and various models for the dust distribution has shown that the standard MW and LMC extinction laws are not suitable for describing the reddening of radiation from extended regions. This result is valid within the limits of our models, which do not represent an exhaustive list of all the possible dust configurations. However, the simplified systems we discuss in the text represent extreme situations which probably bracket many real cases.

Independent of metallicity (LMC-like or MW-like), the UV slopes follow a common trend as a function of the extinction obtained from the Balmer line ratio. This result does not support the idea that the shape of the extinction law depends on the metallicity of the galaxy. In fact, in this case, metal-poor galaxies are expected to be redder than metal-rich galaxies for similar values of the difference between the optical depths of the Balmer lines. There are two interpretations for the lack of a correlation between metallicity and extinction law: (1) a higher metal content in the galaxy increases the number of dust grains, but does not alter the composition or the grain size distribution; (2) geometrical effects dominate, meaning that the inhomogeneity of the dust distribution suppresses differences in the shape of the extinction laws. We cannot disentangle the two interpretations because the two effects, dust composition and geometry, cannot be easily distinguished in the analysis of the radiation from a galaxy.

In order to perform a more detailed comparison between the standard MW and LMC extinction curves and the dust extinction in our galaxies, we have derived the UV and optical extinction law directly from the data. The derivation has been performed under the hypothesis that the dust is a screen in front of the source, which is a simplification, but seems to be suggested by the data themselves. A clumpy distribution of dust is also in agreement with the data, provided that the average number of clumps along the line of sight is $\mathscr{N} \gtrsim 10$. The characteristics of the effective extinction law are, as expected, different from the well-known Milky Way and LMC laws: the overall slope is more gray than the MW or LMC slopes, and, most remarkably, the $2175 \AA$ dust feature is absent within our observational uncertainties.

The shape of the effective extinction law given in equation (25) is clearly a combination of contributions from the chemical and physical properties of the dust grains and from the geometrical distribution of the dust inside galaxies. Disentangling one from the other is difficult, and, therefore, we cannot address basic issues, such as whether or not the dust composition in starburst galaxies is similar to the MW dust composition. However, an "effective extinction law for galaxies" can be still defined, meaning that extended regions of galaxies are characterized by an "average geometrical distribution" of dust, at least for extinction effects in the UV and optical wavelength range.

The effective extinction law probes regions of small optical depth; therefore, it is not strictly related to the total amount of dust in the galaxy. In fact, it is weighted mostly by the regions of lowest extinction, because the flux emerging from highly extincted regions gives virtually no contribution to the total flux. Through equation (25), we can reconstruct the intrinsic spectrum of mildly reddened stellar populations, but no information is given on the heavily reddened stars.

In the derivation of the "effective extinction law" (eq. [25]), we find that the difference between the optical depths of the continuum underlying the Balmer lines, $\tau_{B}^{c}$, is about one-half of the difference between the optical depths of the Balmer emission lines, $\tau_{B}^{l}$. This result has a relatively straightforward interpretation: the ionizing hot stars, which produce the nebular hydrogen, mostly reside in or close to the dusty molecular clouds where they originated, so the nebular lines are affected by the presence of the dusty clouds. On the other hand, the long-lived stars, which contribute to the UV and optical continuum flux, have had the time to drift away from the parent cloud toward regions with smaller amounts of dust. This interpretation is in line with the conclusions reached by Keel (1993) in an analysis of 12 starburst galactic nuceli: "The continuum and emission lines refer to different fractions of the overall stellar population."

The "grayness" of the effective extinction has an interesting application in the case of high-redshift galaxies, for which the UV flux is shifted into optical wavelengths: the UV continuum of galaxies is expected to show a higher flux than the application of the standard extinction laws would predict. This should give a relativly high success rate to those techniques devoted at detecting high-redshift galaxies on the basis of their continuum emission in optical wavebands. Recent works seem to confirm this consideration (cf. Steidel \& Hamilton 1992).

Attempts to determine the dust content in quasars and Seyfert 1 galaxies through the nullification of the $2175 \AA$ dust feature have not yielded results (see, e.g., McKee \& Petrosian 1974; Mushotzky 1984). From our study, it is clear that the effective extinction law in complex environments do not necessarily reproduce the shape observed for the Milky Way stars. The $2175 \AA$ bump does not appear to be a common feature in extragalactic sources. In the case of AGNs, this can be understood as an effect of the high-energy input from the nuclear source into the ISM, which interferes with the process of dust formation (McKee \& Petrosian 1974).

The absorption systems observed in quasar spectra as damped Ly $\alpha$ lines approximate very well the case of a screen in front of a source (the quasar). Therefore, the application of a stellar extinction law seems justified. The detection of reddening in damped Ly $\alpha$ systems has led to efforts to identify the nature of the dust and the dust/gas ratio (e.g., Pei, Fall, \& Bechtold 1991). The overall absence of the $2175 \AA$ dust feature has suggested LMC-like or SMC-like dust. In fact, in the configuration of a dust screen physically detached from the source of radiation, the scattering of photons into the line of sight gives a negligible contribution to the emerging flux, and the absence of the $2175 \AA$ dust feature cannot be attributed to geometry. However, if we speculate that the absence of the 
$2175 \AA$ dust feature in our effective extinction law is due to a dust composition different from the known MW, LMC, or SMC cases, then the presence or absence of the $2175 \AA$ dust feature may not identify the nature of dust in young galaxies.

Although the study of UV and optical spectra of galaxies represents a first important step toward the understanding of the effects of dust extinction in complex environments, it provides a limited view of such effects. Only when IR and radio data will be available for our galaxies we will be able to generalize the approach presented in this work, by measuring the actual dust content of the galaxies and by obtaining a clearer picture of the relative importance of geometry and chemical composition of dust in determining the shape of the effective extinction law.
The authors thank Tim Heckman, Nino Panagia, Ralph Bohlin, and Mike Fall for many useful suggestions and discussions. Special thanks go to Richard White for suggesting the approximation given in equation (14) for the anisotropic scattering and to Claus Leitherer for giving us access to some of the results of the new spectral synthesis code he has developed in collaboration with Carmelle Robert.

The authors acknowledge support from the NASA grant NAG 5-1143 and from the STScI Director Discretionary Research Fund.

T. S. B. acknowledges support from the Brazilian Institutions CNP $_{q}$, CAPES and FAPERGS. A. L. K. would like to thank $\mathrm{CNP}_{\mathrm{q}}$ for support for a collaborative visit to the Departamento de Astronomia, IF-UFRGS.
Bica, E. 1988, A\&A, 195, 76

Bouchet, P., Lequeux, J., Maurice, E., Prevot, L., \& Prevot-Burnichon, M. L. 1985, A\&A, 149, 330

Bromage, G. E., \& Nandy, K. 1983, MNRAS, 204, 29P

Bruzual, A., \& Charlot, S. 1993, ApJ, 405, 538

Bruzual, A., Magris, G. C., \& Calvet, N. 1988, ApJ, 333, 673

Burstein, D., \& Heiles, C. 1982, AJ, 87, 1165

Calzetti, D., Kinney, A. L., Storchi-Bergmann, T., \& Panagia, N. 1993, BAAS, 25,841

Campbell, A. W. 1988, ApJ, 335, 644

Campbell, A. W., Terlevich, R., \& Melnick, J. 1986, MNRAS, 223, 811

Caplan, J., \& Deharveng, L. 1986, A\&A, 155, 297

Charlot, S., \& Fall, S. M. 1993, ApJ, 415, 580

de Jong, T., \& Brink, K. 1986, in Star Formation in Galaxies, ed. C. J. Lonsdale Persson (NASA-CP 2466), 323

Désert, F.-X., Boulanger, F., \& Puget, J. L. 1990, A\&A, 237, 215

Disney, M., Davies, J., \& Phillips, S. 1989, MNRAS, 239, 939

Dopita, M. A., \& Evans, I. N. 1986, ApJ, 307, 431

Draine, B. T., \& Lee, H. M. 1984, ApJ, 285, 89

Fanelli, M. N., O'Connell, R. W., \& Thuan, T. X. 1988, ApJ, 334, 665

Fitzpatrick, E. L. 1986, AJ, 92, 1068

. 1989, in IAU Symp. 135, Interstellar Dust, ed. L. J. Allamandola \& A. G. G. M. Tielens (Dordrecht: Kluwer), 37

Fitzpatrick, E. L., \& Massa, D. 1986, ApJ, 307, 286

. 1988, ApJ, 328, 734

Garmany, C. D., Conti, P. S., \& Chiosi, C. 1982, ApJ, 263, 777

Greenberg, J. M., \& Chlewicki, G. 1983, ApJ, 272, 563

Howarth, I. D. 1983, MNRAS, 203, 301

Hunter, D. A., Gallagher, J. S., III, Rice, W. L., \& Gillett, F. C. 1989, ApJ, 336, 152

Israel, F. P. 1978, A\&A, 70, 769

Israel, F. P., \& Kennicutt, R. L. 1980, Ap. Lett., 21,

Keel, W. C. 1987, A\&A, 172, 43

. 1993, in Massive Stars: Their Lives in the Interstellar Medium, ed. J. P. Cassinelli \& E. B. Churchwell (ASP Conf. Series, 35), 498

Keel, W. C., Kennicutt, R. C., Hummel, E., \& van der Hulst, J. M. 1985, AJ, 90, 708

Kennicutt, R. C. 1991, in Massive Stars in Starbursts, ed. C. Leitherer, N. R., Walborn, T. M., Keckman \& C. A. Norman (Cambridge: Cambridge Univ. Press), 157

Kinney, A. L., Bohlin, R. C., Calzetti, D., Panagia, N., \& Wyse, R. F. G. 1993, ApJS, 86, 5

Kinney, A. L., Bohlin, R. C., \& Neill, J. D. 1991, PASP, 103, 665

Kinney, A. L., Calzetti, D., \& Storchi-Bergmann, T. 1994a, in preparation

Kinney, A. L., Calzetti, D., Storchi-Bergmann, T., \& Bica, E. L. 1994b, ApJ, in press

Koornneef, J., \& Code, A. D. 1981, ApJ, 247, 860

Leisawitz, D., \& Hauser, M. G. 1988, ApJ, 332, 954

\section{REFERENCES}

Leitherer, C. 1990, ApJS, 73, 1

Lequeux, J. 1988, in Dust in the Universe, ed. M. E. Bailey \& D. A. Williams (Cambridge: Cambridge Univ. Press), 449

Lequeux, J., Maucherat-Joubert, M., Deharveng, J.-M., \& Kunth, D. 1981, A\&A, 103, 305

Mathis, J. S. 1983, ApJ, 267, 119

Mathis, J. S., Rumpl, W., \& Nordsieck, K. H. 1977, ApJ, 217, 425

McCall, M. L. 1984, MNRAS, 208, 253

McCall, M. L., Rybski, P. M., \& Shields, G. A. 1985, ApJS, 57, 1

McGaugh, S. S. 1991, ApJ, 380, 140

McKee, C. F., \& Petrosian, V. 1974, ApJ, 189, 17

McQuade, K. A., Kinney, A. L., \& Calzetti, D. 1994, in preparation

Mezger, P. G., \& Smith, L. F. 1977, in IAU Symp. 75, Star Formation, ed. T. de Jong \& A. Maeder (Dordrecht: Reidel), 133

Mushotzsky, R. 1984, in Future of Ultraviolet Astronomy Based on Six Years of IUE Research, ed. J. M. Mead, R. D. Chapman, \& Y. Kondo (NASA CP-2349), 42

Natta, A., \& Panagia, N. 1984, ApJ, 287, 228

Neufeld, D. A. 1990, ApJ, 350, 216

Osterbrock, D. E. 1989, Astrophysics of Gaseous Nebulae and Active Galactic Nuclei (Mill Valley, CA: Univerity Science Books)

Pagel, B. E. J., Edmunds, M. G., Blackwell, D. E., Chun, M. S., \& Smith, G. 1979, MNRAS, 189,95

Pei, Y. C. 1992, ApJ, 395, 130

Pei, Y. C., Fall, S. M., \& Bechtold, J. 1991, ApJ, 395, 130

Peña, M., Ruiz, M. T., \& Maza, J. 1991, A\&A, 251, 417

Rosa, M., Joubert, M., \& Benvenuti, P. 1984, A\&AS, 57, 361

Roy, J.-R., Belley, J., \& Walsh, J. R. 1989, AJ, 97, 1010

Sarazin, C. L. 1976, ApJ, 208, 323

Seaton, M. J. 1979, MNRAS, 187, 73P

Shields, G. A., \& Tinsley, B. M. 1976, ApJ, 203, 66

Skillman, E. D., \& Israel, F. P. 1988, A\&A, 203, 226

Sobolev, V. V. 1983, A Treatise on Radiative Transfer (New York: Van Nostrand)

Steidel, C. C., \& Hamilton, D. 1992, AJ, 104, 941

Storchi-Bergmann, T., Calzetti, D., \& Kinney, A. L. 1994a, ApJ, in press

Storchi-Bergmann, T., Kinney, A. L., \& Challis, P. 1994b, in preparation

Terlevich, R., \& Melnick, J. 1981, MNRAS, 195, 839

Valls-Gabaud, D. 1993, ApJ, 419, 7

van der Hulst, J. M., Kennicutt, R. C., Crane, P. C., \& Rots, A. H. 1988, A\&A, 195,38

Viallefond, F., \& Goss, W. M. 1986, A\&A, 154, 357

Vilchez, J. M., \& Pagel, B. E. J. 1988, MNRAS, 231, 257

Vilchez, J. M., Pagel, B. E. J., Diaz, A. I., Terlevich, E., \& Edmunds, M. G. 1988, MNRAS, 235, 633

Waller, W. H., Gurwell, M., \& Tamura, M. 1992, AJ, 104, 63

White, R. L. 1979, ApJ, 229, 954

Witt, A. N., Thronson, H. A., \& Capuano, J. M. 1992, ApJ, 393, 611 